Pacific

Journal of

Mathematics

\title{
THE EQUIVARIANT CHOW RINGS OF QUOT SCHEMES
}

Tom BRAden, Linda Chen AND Frank SotTILE 


\title{
THE EQUIVARIANT CHOW RINGS OF QUOT SCHEMES
}

\author{
Tom Braden, Linda Chen And Frank Sottile
}

\begin{abstract}
We give a presentation for the (integral) torus-equivariant Chow ring of the quot scheme, a smooth compactification of the space of rational curves of degree $d$ in the Grassmannian. For this presentation, we refine Evain's extension of the method of Goresky, Kottwitz, and MacPherson to express the torus-equivariant Chow ring in terms of the torus-fixed points and explicit relations coming from the geometry of families of torus-invariant curves. As part of this calculation, we give a complete description of the torusinvariant curves on the quot scheme and show that each family is a product of projective spaces.
\end{abstract}

\section{Introduction}

Let $k$ be an algebraically closed field and let $d, n, r$ be nonnegative integers with $r<n$. We study the quot scheme $2_{d}:=2_{d}(r, n)$ parametrizing quotient sheaves on $\mathbb{P}^{1}$ of the trivial vector bundle $\mathbb{O}_{\mathbb{P} 1}^{n}$ which have rank $r$ and degree $d$. When $r>0$, this is a compactification of the space $\mu_{d}$ of parametrized rational curves of degree $d$ on the Grassmannian $G(r, n)$ of $r$-dimensional quotients of $k^{n}$. Indeed, a morphism from $\mathbb{P}^{1}$ to $G(r, n)$ of degree $d$ is equivalent to a quotient bundle $\mathrm{O}_{\mathbb{P} 1}^{n} \rightarrow \mathcal{T}$ of rank $r$ and degree $d$.

Strømme [1987] showed that $\mathscr{2}_{d}(r, n)$ is a smooth, projective, rational variety of dimension $r(n-r)+n d$. He described the decomposition into Białynicki-Birula cells induced by an action of a one-dimensional torus on $2_{d}$, thereby determining its Betti numbers. He also gave a presentation of its integral Chow ring (his Theorem 5.3) in terms of generators and relations. However, the set of generators is far from minimal, and the relations are given by the annihilator of a certain class, and are therefore nonexplicit. He also gave a more elementary set of generators for its rational Chow ring.

Later, Bertram [1997] used the geometry of $2_{d}$ to determine the (small) quantum cohomology ring of the Grassmannian. He used a recursive description of the

MSC2000: 55N91, 14M15, 14F43, $14 \mathrm{C} 05$.

Keywords: equivariant cohomology, Chow ring, quot scheme, Grassmannian.

Braden supported in part by NSF grant DMS-0201823. Chen supported in part by NSF VIGRE grant DMS-9810750 and NSF grant DMS-0432701. Sottile supported in part by NSF CAREER grant DMS-0134860 and the Clay Mathematical Institute. 
boundary $2_{d} \backslash M_{d}$ to show that the 3-point genus zero Gromov-Witten invariants of the Grassmannian are equal to particular intersection numbers on $2_{d}$. By studying certain types of intersections on quot schemes, he obtained a quantum Schubert calculus for the Grassmannian. However, he did not need to compute the full cohomology ring of $2_{d}$.

Our main result is a presentation of the $T$-equivariant Chow ring $A_{T}^{*}\left(2_{d}\right)$ for an action of the torus

$$
T=T_{k^{n}} \times T_{\mathbb{P} 1}
$$

on $2_{d}$, where $T_{k^{n}}=\left(\mathbb{G}_{m}\right)^{n}$ acts diagonally on $k^{n}$ and $T_{\mathbb{P}^{1}}=\mathbb{G}_{m}$ acts primitively on $\mathbb{P}^{1}$. Since the ordinary Chow ring $A^{*}\left(2_{d}\right)$ is the quotient of $A_{T}^{*}\left(2_{d}\right)$ by the image of $A_{T}^{>0}(p)$, this also determines $A^{*}\left(2_{d}\right)$. When $k=\mathbb{C}$, the cycle class map induces an isomorphism between Chow and cohomology rings, so our result also determines the $T$-equivariant and ordinary cohomology rings of $2_{d}$.

Our presentation gives $A_{T}^{*}\left(2_{d}\right)$ as an explicit subring of a direct sum of polynomial rings. This arises from an analysis of the localization map. When an algebraic torus $T$ acts on a smooth variety $X$ with a finite fixed point set $X^{T}$, the inclusion $i: X^{T} \hookrightarrow X$ induces the localization map of (integral) equivariant Chow rings

$$
i^{*}: A_{T}^{*}(X) \rightarrow A_{T}^{*}\left(X^{T}\right)=\bigoplus_{p \in X^{T}} A_{T}^{*}(p) .
$$

Each summand $A_{T}^{*}(p)$ is canonically isomorphic to the symmetric algebra $S$ of the character group of $T$. If $X$ is projective, then $i^{*}$ is injective, so computing the ring $A_{T}^{*}(X)$ reduces to computing the image of $i^{*}$.

When $k=\mathbb{C}$, one can consider the corresponding localization map for rational equivariant cohomology. In this case, a result of Chang and Skjelbred [1974] implies that the image of the localization map is cut out by the images of the $T$-equivariant cohomology of components of the one-skeleton of $X$ : the set of points that are fixed by some codimension one subtorus of $T$. These components are closures of families of $T$-invariant curves. In particular, when $X$ has finitely many one-dimensional $T$-orbits (whose closures are $T$-invariant curves), Goresky, Kottwitz, and MacPherson [1998] used this to describe the image of the localization map for equivariant cohomology. Each $T$-invariant curve gives a relation, and these GKM relations cut out the image. Brion [1997] showed that this remains true for rational equivariant Chow rings of varieties over any algebraically closed field.

Our action of $T$ on $2_{d}$ has finitely many fixed points, but there are infinitely many $T$-invariant curves. The GKM relations remain valid, but are now insufficient to cut out the image of the localization map, even over $\mathbb{Q}$; there will be extra relations coming from connected components of the one-skeleton of $2_{d}$. Brion [1997] adapted the result of Chang and Skjelbred to Chow groups, showing that the relations given by these families are sufficient to cut out $A_{T}^{*}(X)_{\mathbb{Q}}$. Determining 
these relations explicitly is more difficult than for the GKM relations, however, and few cases have been worked out in detail.

One case for which the relations are known is when $X$ is a Hilbert scheme of points on a toric surface, which has families of $T$-invariant curves. Following a suggestion of Brion, Evain [2007] used Edidin and Graham's [1998b] version of the Atiyah-Bott-Berline-Vergne localization formula for equivariant Chow groups to give relations in terms of ideal-membership. The relations come from elements of the $T$-equivariant Chow rings of the families of $T$-invariant curves.

We discuss this in Section 5, and give a more explicit formula for Evain's relations which holds when each component $Y$ of $X^{T^{\prime}}$ for $T^{\prime} \subset T$ of codimension one has smooth $T$-invariant subvarieties $Z$ whose classes [Z] generate $A_{T}^{*}(Y)$. We derive necessary and sufficient linear relations over $\mathbb{Q}$ from Evain's idealmembership relations. Lastly, we show that if the $T$-weights of the tangent space at each fixed point are not too dependent (see Theorem 5.5), then Evain's relations also determine the integral Chow ring.

All these additional hypotheses hold for $2_{d}$. In fact, the components $Y$ we get are quite simple: all are products of projective spaces. As a result, we obtain explicit descriptions of the equivariant Chow ring of $2_{d}$, both rationally and integrally.

To describe the combinatorics of fixed points in $2_{d}^{T}$, we use the following notations. For an element $\mathbf{a}=\left(a_{1}, \ldots, a_{n}\right) \in \mathbb{Z}^{n}$, we define $|\mathbf{a}|=\sum_{i} a_{i}$. We use addition and subtraction on $\mathbb{Z}^{n}$ considered as an abelian group, and denote the identity element by $\mathbf{0}=(0, \ldots, 0)$. If $\mathbf{a} \in\left(\mathbb{Z}_{\geq 0}\right)^{n}$, we set $\mathbf{a} !=\prod_{i} a_{i} !$, where $0 !=1$. Finally, we use the partial order $\mathbf{a} \leq \mathbf{b}$ to mean $a_{i} \leq b_{i}$ for all $i$.

In Section 2, we give an explicit parametrization of the fixed point set $2_{d}^{T}$ by a set $\mathscr{F}$ of triples $(\boldsymbol{\delta}, \mathbf{a}, \mathbf{b})$, where

- $\delta \in\{0,1\}^{n}$ takes the value 1 exactly $n-r$ times, so that $|\delta|=n-r$, and

- a, $\mathbf{b}$ are elements of $\left(\mathbb{Z}_{\geq 0}\right)^{n}$ which satisfy $|\mathbf{a}|+|\mathbf{b}|=d$ and for which $\delta_{i}=0$ implies $a_{i}=0$ and $b_{i}=0$.

The fixed points are maximally degenerate quotient sheaves supported at 0 and $\infty$; the data $\mathbf{a}$ and $\mathbf{b}$ describe the structure of the stalks as modules over $O_{\mathbb{P}^{1}}$ and as representations of $T$.

Recall that $A_{T}^{*}(p)=S$, the symmetric algebra of the character group of $T$. We have $S=\mathbb{Z}\left[\mathbf{e}_{1}, \ldots, \mathbf{e}_{n}, \mathbf{f}\right]$, where $\mathbf{e}_{1}, \ldots, \mathbf{e}_{n}$, and $\mathbf{f}$ are dual to the obvious basis coming from the decomposition $T=T_{k^{n}} \times T_{\mathbb{P} 1}$. In particular, $\mathbf{f}$ restricts to the identity character on $T_{\mathbb{P} 1}$ and to the trivial character of $T_{k^{n}}$. We write $S_{\mathbb{Q}}$ for $S \otimes_{\mathbb{Z}} \mathbb{Q}$ and sometimes $S_{\mathbb{Z}}$ for $S$, when we wish to emphasize our ring of scalars. We write $S^{\mathscr{F}}$ for the set of tuples of polynomials $\left(f_{(\boldsymbol{\delta}, \mathbf{a}, \mathbf{b})} \in S \mid(\boldsymbol{\delta}, \mathbf{a}, \mathbf{b}) \in \mathscr{F}\right)$. Then $S^{\mathscr{F}}=A_{T}^{*}\left(Q_{d}^{T}\right)$, under the identification of $\mathscr{F}_{F}$ with $Q_{d}^{T}$. We exhibit the image of the localization map as a subring of $S^{\mathscr{F}}$. 
In Section 3 we describe a finite set of $T$-invariant curves which span the tangent space at each fixed point. In Section 4, we describe the families of $T$-invariant curves and their closures. Based on this description, our relations for the image of $i_{2_{d}}^{*}$ are

I For any pair $(\boldsymbol{\delta}, \mathbf{a}, \mathbf{b}),\left(\boldsymbol{\delta}^{\prime}, \mathbf{a}^{\prime}, \mathbf{b}^{\prime}\right) \in \mathscr{F}$ with $\boldsymbol{\delta}=\boldsymbol{\delta}^{\prime}, \mathbf{a}=\mathbf{a}^{\prime}$ and $\mathbf{b}=\mathbf{b}^{\prime}$ except in positions $i$ and $j$, and $\delta_{i}=\delta_{j}^{\prime}=1$ and $\delta_{j}=\delta_{i}^{\prime}=0$, we have

$$
f_{(\boldsymbol{\delta}, \mathbf{a}, \mathbf{b})} \equiv f_{\left(\boldsymbol{\delta}^{\prime}, \mathbf{a}^{\prime}, \mathbf{b}^{\prime}\right)} \quad \bmod \mathbf{e}_{j}-\mathbf{e}_{i}+\left(a_{j}^{\prime}-a_{i}\right) \mathbf{f} .
$$

(Note that $\left(a_{j}^{\prime}-a_{i}\right)=-\left(b_{j}^{\prime}-b_{i}\right)$, since $a_{i}+b_{i}=a_{j}^{\prime}+b_{j}^{\prime}$.)

II (a) For any pair $(\boldsymbol{\delta}, \mathbf{a}, \mathbf{b}),\left(\boldsymbol{\delta}, \mathbf{a}^{\prime}, \mathbf{b}\right) \in \mathscr{F}$ with $\mathbf{a}, \mathbf{a}^{\prime}$ agreeing except in positions $i$ and $j$, we have

$$
f_{(\boldsymbol{\delta}, \mathbf{a}, \mathbf{b})} \equiv f_{\left(\boldsymbol{\delta}, \mathbf{a}^{\prime}, \mathbf{b}\right)} \quad \bmod \mathbf{e}_{j}-\mathbf{e}_{i}+\left(a_{j}^{\prime}-a_{i}\right) \mathbf{f} .
$$

(b) For any pair $(\boldsymbol{\delta}, \mathbf{a}, \mathbf{b}),\left(\boldsymbol{\delta}, \mathbf{a}, \mathbf{b}^{\prime}\right) \in \mathscr{F}$ with $\mathbf{b}, \mathbf{b}^{\prime}$ agreeing except in positions $i$ and $j$, we have

$$
f_{(\boldsymbol{\delta}, \mathbf{a}, \mathbf{b})} \equiv f_{\left(\boldsymbol{\delta}, \mathbf{a}, \mathbf{b}^{\prime}\right)} \quad \bmod \mathbf{e}_{j}-\mathbf{e}_{i}+\left(b_{i}-b_{j}^{\prime}\right) \mathbf{f} .
$$

(c) If we have $(\boldsymbol{\delta}, \mathbf{a}, \mathbf{b}),\left(\boldsymbol{\delta}, \mathbf{a}^{\prime}, \mathbf{b}\right),\left(\boldsymbol{\delta}, \mathbf{a}, \mathbf{b}^{\prime}\right) \in \mathscr{F}^{\mathscr{F}}$ satisfying both of the previous conditions (with the same $i$ and $j$ ), and in addition $a_{j}^{\prime}-a_{i}=b_{i}-b_{j}^{\prime}$, then $D f_{(\boldsymbol{\delta}, \mathbf{a}, \mathbf{b})}-D f_{\left(\boldsymbol{\delta}, \mathbf{a}^{\prime}, \mathbf{b}\right)}-D f_{\left(\boldsymbol{\delta}, \mathbf{a}, \mathbf{b}^{\prime}\right)}+D f_{\left(\boldsymbol{\delta}, \mathbf{a}^{\prime}, \mathbf{b}^{\prime}\right)} \equiv 0 \quad \bmod \mathbf{e}_{j}-\mathbf{e}_{i}+\left(a_{j}^{\prime}-a_{i}\right) \mathbf{f}$,

where $D$ is the differentiation in the direction of $\mathbf{e}_{j}^{\vee}$, the dual basis vector to $\mathbf{e}_{j}$.

(c)' Under the hypotheses of II(c),

$$
f_{(\boldsymbol{\delta}, \mathbf{a}, \mathbf{b})}-f_{\left(\boldsymbol{\delta}, \mathbf{a}^{\prime}, \mathbf{b}\right)}-f_{\left(\boldsymbol{\delta}, \mathbf{a}, \mathbf{b}^{\prime}\right)}+f_{\left(\boldsymbol{\delta}, \mathbf{a}^{\prime}, \mathbf{b}^{\prime}\right)} \equiv 0 \quad \bmod \left(\mathbf{e}_{j}-\mathbf{e}_{i}+\left(a_{j}^{\prime}-a_{i}\right) \mathbf{f}\right)^{2} .
$$

III For every $(\boldsymbol{\delta}, \mathbf{a}, \mathbf{b}) \in \mathscr{F}$ and every $\mathbf{0} \leq \mathbf{b}^{\prime}<\mathbf{b}$,

$$
\sum_{\mathbf{b}^{\prime} \leq \mathbf{c} \leq \mathbf{b}} \frac{(-1)^{|\mathbf{c}|}}{(\mathbf{b}-\mathbf{c}) !\left(\mathbf{c}-\mathbf{b}^{\prime}\right) !} D^{|\mathbf{b}|-\left|\mathbf{b}^{\prime}\right|-1} f_{(\delta, \mathbf{a}+\mathbf{c}, \mathbf{b}-\mathbf{c})} \equiv 0 \quad \bmod \mathbf{f},
$$

where $D$ is the differentiation in the direction of $\mathbf{f}^{\vee}$, the dual basis vector to $\mathbf{f}$.

III' For every $(\boldsymbol{\delta}, \mathbf{a}, \mathbf{b}) \in \mathscr{F}, \mathbf{b} \neq \mathbf{0}$,

$$
\sum_{0 \leq \mathbf{c} \leq \mathbf{b}} \frac{(-1)^{|\mathbf{c}|}}{\mathbf{c} !(\mathbf{b}-\mathbf{c}) !} f_{(\boldsymbol{\delta}, \mathbf{a}+\mathbf{c}, \mathbf{b}-\mathbf{c})} \equiv 0 \quad \bmod \mathbf{f}^{|\mathbf{b}|} .
$$

By this, we mean that the left-hand side, which a priori is an element of $S_{\mathbb{Q}}$, actually lies in $\mathbf{f}^{\mathbf{b} \mid} S_{\mathbb{Z}}$. 
Relations I, II(a), and II(b) are standard GKM relations, while the rest come from families of $T$-invariant curves. In particular, relations $\mathbf{I I}(\mathrm{c}) / \mathbf{I I}(\mathrm{c})^{\prime}$ (respectively III/III') come from certain families whose closures are isomorphic to $\mathbb{P}^{1} \times \mathbb{P}^{1}$ (respectively arbitrary products of projective spaces).

Theorem 1.1. The rational equivariant Chow ring $A_{T}^{*}\left(2_{d}\right)_{\mathbb{Q}}$ is isomorphic to the set of tuples $f=\left(f_{(\boldsymbol{\delta}, \mathbf{a}, \mathbf{b})}\right) \in S_{\mathbb{Q}}^{\mathscr{F}}$ subject to the relations $\mathbf{I}, \mathbf{I I}(\mathrm{a})-(\mathrm{c})$, and $\mathbf{I I I}$.

The integral equivariant Chow ring $A_{T}^{*}\left(2_{d}\right)$ is isomorphic to the set of tuples $f=\left(f_{(\boldsymbol{\delta}, \mathbf{a}, \mathbf{b})}\right) \in S_{\mathbb{Z}}^{\mathscr{F}}$ subject to the relations $\mathbf{I}, \mathbf{I I}(\mathrm{a})(\mathrm{b})(\mathrm{c})^{\prime}$, and $\mathbf{I I I}^{\prime}$.

We prove Theorem 1.1 in Section 5E. Since the equivariant Chow ring determines the ordinary Chow ring for smooth spaces, this gives in principle a complete description of the Chow ring of $2_{d}$. The resulting computation of Betti numbers is the same as Strømme's computation.

Strømme's generators of the rational cohomology ring of $2_{d}$ were Künneth components of the Chern classes of the tautological vector bundle $\mathscr{S}$ on $\mathbb{P}^{1} \times \mathscr{2}_{d}$ defined by the universal exact sequence

$$
0 \rightarrow \mathscr{Y} \rightarrow \mathrm{O}_{\mathbb{P}^{1} \times \mathscr{2}_{d}}^{n} \rightarrow \mathscr{T} \rightarrow 0
$$

where the restriction of $\mathscr{T}$ to $\mathbb{P}^{1} \times\{p\} \cong \mathbb{P}^{1}$ is the quotient sheaf of $\mathbb{O}_{\mathbb{p} 1}^{n}$ represented by $p \in 2_{d}$. In Section 6, we describe the equivariant Chern classes of $\mathscr{Y}$ in $A_{T}^{*}\left(2_{d}\right)$ and thus lifts of Strømme's generators to the equivariant Chow groups. In Section 7 we work this out explicitly for $\mathscr{2}_{2}(0,2)$, using Theorem 1.1 to describe the equivariant and ordinary Chow rings and giving explicit lifts of Strømme's generators as localized classes.

\section{Torus-fixed points of $2_{d}$}

Let $\mathbf{e}_{1}, \ldots, \mathbf{e}_{n}$ denote the standard basis of $k^{n}$. Write $T_{k^{n}}$ for the group of diagonal matrices in this basis. Let $[x, y]$ be coordinates on $\mathbb{P}^{1}$ with $x$ vanishing at 0 and $y$ at $\infty$. For $T_{\mathbb{P}^{1}}$ acting on $\mathbb{P}^{1}$ with fixed points 0 and $\infty$, the torus $T:=T_{k^{n}} \times T_{\mathbb{P} 1}$ acts on $2_{d}$ naturally as indicated by the given splitting.

The $T$-fixed points are indexed by triples $(\boldsymbol{\delta}, \mathbf{a}, \mathbf{b})$ in the set $\mathscr{F}$ of Section 1 . The fixed point corresponding to $(\boldsymbol{\delta}, \mathbf{a}, \mathbf{b})$ is the sequence of sheaves on $\mathbb{P}^{1}$,

$$
\mathscr{S}_{(\boldsymbol{\delta}, \mathbf{a}, \mathbf{b})} \rightarrow \mathcal{O}_{\mathbb{P} 1}^{n} \rightarrow \mathscr{T}_{(\boldsymbol{\delta}, \mathbf{a}, \mathbf{b})},
$$

where $\mathscr{Y}_{(\boldsymbol{\delta}, \mathbf{a}, \mathbf{b})}$ is the image of the map

$$
\bigoplus_{i=1}^{n} \mathcal{O}_{\mathbb{P} 1}\left(-a_{i}-b_{i}\right) \stackrel{\operatorname{diag}\left(\delta_{i} x^{a_{i}} y^{b_{i}}\right)}{\longrightarrow} \mathcal{O}_{\mathbb{P} 1}^{n} .
$$

We identify this fixed point with the subsheaf $\mathscr{Y}_{(\boldsymbol{\delta}, \mathbf{a}, \mathbf{b})}$ of $\mathbb{O}_{\mathbb{P} 1}^{n}$. 
We introduce the following notation. For natural numbers $a, b$, let $\mathscr{T}_{a, b}$ be the subsheaf of $\mathcal{O}_{\mathbb{p} 1}$ which is the image of the map

$$
\mathcal{O}_{\mathbb{P} 1}(-a-b) \stackrel{x^{a} y^{b}}{\longrightarrow} \mathcal{O}_{\mathbb{P}^{1}} .
$$

Under the identification of modules over $O_{\mathbb{P}^{1}}$ with saturated graded modules of the homogeneous coordinate ring $k[x, y], \mathscr{S}_{a, b}$ is the ideal of $k[x, y]$ generated by $x^{a} y^{b}$. The quotient $\mathrm{O}_{\mathbb{p}^{1}} / \mathscr{S}_{a, b}$ is the skyscraper sheaf

$$
\mathscr{T}_{a, b}=\mathcal{O}_{\mathbb{P}^{1}} / \mathfrak{m}_{0}^{a} \oplus \mathcal{O}_{\mathbb{P}^{1}} / \mathfrak{m}_{\infty}^{b}
$$

on $\mathbb{P}^{1}$ supported at 0 and at $\infty$. Here, $\mathfrak{m}_{p}$ is the sheaf of ideals cutting out the point $p \in \mathbb{P}^{1}$. Then

$$
\begin{aligned}
& \mathscr{T}_{(\boldsymbol{\delta}, \mathbf{a}, \mathbf{b})}=\bigoplus_{\delta_{i}=1} \mathscr{T}_{a_{i}, b_{i}} \cdot \mathbf{e}_{i}, \quad \text { and } \\
& \mathscr{T}_{(\boldsymbol{\delta}, \mathbf{a}, \mathbf{b})}=\bigoplus_{\delta_{j}=1} \mathscr{T}_{a_{j}, b_{j}} \cdot \mathbf{e}_{j} \oplus \bigoplus_{\delta_{j}=0} \mathcal{O}_{\mathbb{P} 1} \cdot \mathbf{e}_{j} .
\end{aligned}
$$

In the sum, $\delta_{j}=0$ means those $j$ in $\{1, \ldots, n\}$ with $\delta_{j}=0$, and the same for $\delta_{j}=1$.

The tangent space to $2_{d}$ at this fixed point is

$$
\operatorname{Hom}\left(\mathscr{Y}_{(\boldsymbol{\delta}, \mathbf{a}, \mathbf{b})}, \mathscr{T}_{(\boldsymbol{\delta}, \mathbf{a}, \mathbf{b})}\right) .
$$

Let $\hat{\mathbf{e}}_{1}, \ldots, \hat{\mathbf{e}}_{n}$ be the basis dual to $\mathbf{e}_{1}, \ldots, \mathbf{e}_{n}$. For each $i, j$, set

$$
\mathbf{E}_{i j}:=\hat{\mathbf{e}}_{i} \otimes \mathbf{e}_{j} \in \operatorname{Hom}\left(k^{n}, k^{n}\right) .
$$

These $\mathbf{E}_{i j}$ form a basis for $\operatorname{Hom}\left(k^{n}, k^{n}\right)$.

Theorem 2.1. The tangent space $T_{(\boldsymbol{\delta}, \mathbf{a}, \mathbf{b})} \mathscr{2}_{d}$ is canonically identified with

$$
\bigoplus_{\delta_{i}=1} \bigoplus_{\delta_{j}=0} \operatorname{Hom}\left(\mathscr{S}_{a_{i}, b_{i}}, \mathcal{O}_{\mathbb{P}^{1}}\right) \cdot \mathbf{E}_{i j} \oplus \bigoplus_{\delta_{i}=1} \bigoplus_{\delta_{j}=1} \operatorname{Hom}\left(\mathscr{Y}_{a_{i}, b_{i}}, \mathscr{T}_{a_{j}, b_{j}}\right) \cdot \mathbf{E}_{i j}
$$

We now give $T$-bases (bases of $T$-eigenvectors) for these summands and determine the corresponding weights. Fix a basis for the character group of $T$ as follows. Extend the action of $T_{k^{n}}$ on $O_{\mathbb{P} 1}^{n}$ to $T$ by letting the factor $T_{\mathbb{P}^{1}}$ act trivially. Then we abuse notation and denote the character of $T$ acting on the $i$-th basis vector $\mathbf{e}_{i}$ by the same symbol $\mathbf{e}_{i}$. Thus the dual basis element $\hat{\mathbf{e}}_{i}$ has $T$-weight $-\mathbf{e}_{i}$.

Similarly, extend the action of $T_{\mathbb{P}^{1}}$ on $\mathbb{P}^{1}$ to an action of $T$ by letting $T_{k^{n}}$ act trivially, and denote by $\mathbf{f}$ the character of $T$ corresponding to the action on the dense orbit $\mathbb{P}^{1} \backslash\{0, \infty\}$. More precisely, we can let $T_{\mathbb{P}^{1}} \cong \mathbb{C}^{*}$ act on the homogeneous coordinates $k[x, y]$ of $\mathbb{P}^{1}$ by $q \cdot x=q x$ and $q \cdot y=y$. Thus $T$ acts on the rational function $z:=x / y$ with weight $\mathbf{f}$, and on the monomial $z^{a}=x^{a} y^{-a}$ with weight $a \mathbf{f}$. 
The first sum of (2-1) involves spaces of the form $\operatorname{Hom}\left(\mathscr{S}_{a, b}, \mathcal{O}_{\mathbb{P} 1}\right)=H^{0}\left(\mathscr{S}_{a, b}^{*}\right)$. This space of sections has a monomial $T$-basis

$$
\left\{z^{c} \mid-a \leq c \leq b\right\} .
$$

For example, $H^{0}\left(\mathscr{Y}_{1,2}^{*}\right)=k \cdot\left\{z^{-1}, z^{0}=1, z, z^{2}\right\}$. Thus if $\delta_{i}=1$ and $\delta_{j}=0$, then the piece $\operatorname{Hom}\left(\mathscr{S}_{a_{i}, b_{i}}, \hat{O}_{\mathbb{P} 1}\right) \cdot \mathbf{E}_{i j}$ of the tangent space has a monomial $T$-basis $z^{c} \cdot \mathbf{E}_{i j}$ for all $-a_{i} \leq c \leq b_{i}$. The basis element $z^{c} \cdot \mathbf{E}_{i j}$ has $T$-weight

$$
\mathbf{e}_{j}-\mathbf{e}_{i}+c \mathbf{f} .
$$

The second sum of (2-1) involves spaces of the form $\operatorname{Hom}\left(\mathscr{S}_{a, b}, \mathscr{T}_{\alpha, \beta}\right)$. Since $\mathscr{T}_{\alpha, \beta}$ is a skyscraper sheaf supported at 0 and $\infty$, a map $\phi \in \operatorname{Hom}\left(\mathscr{T}_{a, b}, \mathscr{T}_{\alpha, \beta}\right)$ is determined by its actions at 0 and at $\infty$. At $0, z=x / y$ is a local parameter, so the map $\phi$ becomes

$$
\phi: z^{a} \mathbb{C}[z] \rightarrow \mathbb{C}[z] /\left\langle z^{\alpha}\right\rangle,
$$

and thus has the form $z^{-a} f(z)$ where $f(z)$ has degree less than $\alpha$. At $\infty, z^{-1}$ is a local parameter, and the map $\phi$ has the form $z^{b} g\left(z^{-1}\right)$ where $g(z)$ is a polynomial of degree less than $\beta$. Thus $\operatorname{Hom}\left(\mathscr{Y}_{a, b}, \mathscr{T}_{\alpha, \beta}\right)$ has the monomial $T$-basis

$$
k \cdot\left\{z^{(\alpha-c)-a} \mid 1 \leq c \leq \alpha\right\} \oplus k \cdot\left\{z^{b-(\beta-c)} \mid 1 \leq c \leq \beta\right\},
$$

where elements in the first summand act by zero on the stalk at $\infty$, and elements of the second summand act by zero at 0 .

Thus, if $\delta_{i}=\delta_{j}=1$, then the summand $\operatorname{Hom}\left(\mathscr{T}_{a_{i}, b_{i}}, \mathscr{T}_{a_{j}, b_{j}}\right) \cdot \mathbf{E}_{i j}$ of (2-1) has a monomial $T$-basis

$$
\left\{z^{\left(a_{j}-c\right)-a_{i}} \cdot \mathbf{E}_{i j} \mid 1 \leq c \leq a_{j}\right\} \cup\left\{z^{\left(b_{j}-c\right)-b_{i}} \cdot \mathbf{E}_{i j} \mid 1 \leq c \leq b_{j}\right\}
$$

with corresponding $T$-weights

$$
\mathbf{e}_{j}-\mathbf{e}_{i}+\left(\left(a_{j}-c\right)-a_{i}\right) \mathbf{f} \text { and } \mathbf{e}_{j}-\mathbf{e}_{i}+\left(b_{i}-\left(b_{j}-c\right)\right) \mathbf{f} .
$$

We note that this discussion gives a basis for $T_{(\boldsymbol{\delta}, \mathbf{a}, \mathbf{b})} 2_{d}$ consisting of

$$
\begin{aligned}
\sum_{\delta_{j}=0} \sum_{\delta_{i}=1}\left(a_{i}+b_{i}+1\right)+\sum_{\delta_{i}=1} \sum_{\delta_{j}=1}\left(a_{j}+b_{j}\right) & =(n-r) \cdot(d+r)+r \cdot d \\
& =r \cdot(n-r)+n d=\operatorname{dim} 2_{d}
\end{aligned}
$$

elements, which shows that $\mathscr{2}_{d}$ is smooth at the $T$-fixed point $\mathscr{Y}_{(\boldsymbol{\delta}, \mathbf{a}, \mathbf{b})}$, and hence everywhere, since $2_{d}$ is projective. In Section 3 , we will describe $T$-invariant curves in $\mathscr{2}_{d}$ containing $\mathscr{Y}_{(\boldsymbol{\delta}, \mathbf{a}, \mathbf{b})}$ whose tangent directions at $\mathscr{S}_{(\boldsymbol{\delta}, \mathbf{a}, \mathbf{b})}$ coincide with this given $T$-basis of $T_{(\boldsymbol{\delta}, \mathbf{a}, \mathbf{b})} 2_{d}$.

Example 2.2. The quot scheme $2:=\mathscr{2}_{2}(0,2)$ of rank 0 and degree 2 quotients of $\mathrm{O}_{\mathbb{P} 1}^{2}$ has dimension $r(n-r)+d n=2 \cdot 0+2 \cdot 2=4$. Note that the associated 
Grassmannian is a point. Since $r=0$, the index $\delta$ is the same for each fixed point, $\delta_{1}=\delta_{2}=1$, and so the fixed points are indexed by quadruples $\left(a_{1}, a_{2}, b_{1}, b_{2}\right)$ of nonnegative integers whose sum is 2 . Thus there are ten fixed points. We represent the fixed point $\left(a_{1}, a_{2}, b_{1}, b_{2}\right)$ by two columns of boxes superimposed on a horizontal line where the $i$-th column has $a_{i}$ boxes above the horizontal line and $b_{i}$ boxes below it. For example,

$$
\square \leftrightarrow(2,0,0,0), \quad \square \leftrightarrow(1,1,0,0), \quad \text { and } \square \leftrightarrow(1,0,0,1)
$$

The fixed point corresponding to $\square_{\text {in }}$ is the exact sequence of sheaves on $\mathbb{P}^{1}$,

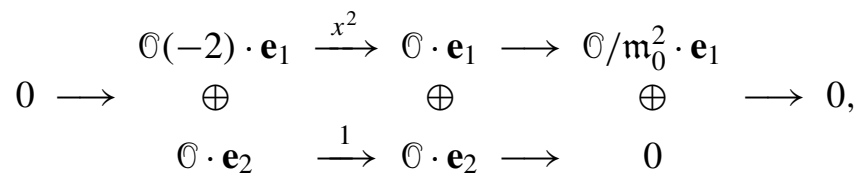

where $O=O_{p^{1}}$. The tangent space at this fixed point is the sum of the two 2dimensional $T$-invariant spaces of homomorphisms having the indicated $T$-bases:

$$
\begin{aligned}
\operatorname{Hom}\left(\mathcal{O}, \mathcal{O} / \mathfrak{m}_{0}^{2}\right) \cdot \mathbf{E}_{21} & =k\{1, z\} \cdot \mathbf{E}_{21}, \\
\operatorname{Hom}\left(x^{2} \mathcal{O}, \mathcal{O} / \mathfrak{m}_{0}^{2}\right) \cdot \mathbf{E}_{11} & =k\left\{z^{-2}, z^{-1}\right\} \cdot \mathbf{E}_{11} .
\end{aligned}
$$

As before, $z:=x / y$ is a local parameter at 0 and $z^{-1}$ is a local parameter at $\infty$. These basis elements have four distinct $T$-weights

$$
\mathbf{e}_{1}-\mathbf{e}_{2}, \mathbf{e}_{1}-\mathbf{e}_{2}+\mathbf{f} \text {, and }-\mathbf{f},-2 \mathbf{f}
$$

The fixed point corresponding to $\square$ is the exact sequence of sheaves on $\mathbb{P}^{1}$,

$$
\begin{aligned}
& \mathcal{O}(-1) \cdot \mathbf{e}_{1} \stackrel{x}{\longrightarrow} \mathcal{O} \cdot \mathbf{e}_{1} \longrightarrow \mathcal{O} / \mathfrak{m}_{0} \cdot \mathbf{e}_{1} \\
& 0 \longrightarrow \oplus \quad \oplus \quad \oplus \quad \longrightarrow 0 . \\
& \mathrm{O}(-1) \cdot \mathbf{e}_{2} \stackrel{y}{\longrightarrow} \mathrm{O} \cdot \mathbf{e}_{2} \longrightarrow \mathrm{O} / \mathfrak{m}_{\infty} \cdot \mathbf{e}_{2}
\end{aligned}
$$

The tangent space at this fixed point is the sum of four 1-dimensional $T$-invariant spaces of homomorphisms having bases and weights as indicated from the following table:

\begin{tabular}{l|rr}
$T$-eigenspace & basis & $T$-weight \\
\hline $\operatorname{Hom}\left(x \mathcal{O}, \mathcal{O} / \mathfrak{m}_{0}\right) \cdot \mathbf{E}_{11}$ & $z^{-1} \cdot \mathbf{E}_{11}$ & $-\mathbf{f}$ \\
$\operatorname{Hom}\left(x \mathscr{O}, \mathcal{O} / \mathfrak{m}_{\infty}\right) \cdot \mathbf{E}_{12}$ & $1 \cdot \mathbf{E}_{12}$ & $\mathbf{e}_{2}-\mathbf{e}_{1}$ \\
$\operatorname{Hom}\left(y \mathcal{O}, \mathcal{O} / \mathfrak{m}_{0}\right) \cdot \mathbf{E}_{21}$ & $1 \cdot \mathbf{E}_{21}$ & $\mathbf{e}_{1}-\mathbf{e}_{2}$ \\
$\operatorname{Hom}\left(y \mathcal{O}, \mathcal{O} / \mathfrak{m}_{\infty}\right) \cdot \mathbf{E}_{22}$ & $z \cdot \mathbf{E}_{22}$ & $\mathbf{f}$
\end{tabular}




\section{3. $T$-invariant curves}

A flat family $\mathscr{S} \rightarrow \mathbb{P}^{1}$ equipped with a $T$-action whose fibre $\mathscr{S}(s, t)$ over a point $[s, t] \in \mathbb{P}^{1}$ is a free subsheaf of $\mathrm{O}_{\mathbb{P} 1}^{n}$ of rank $n-r$ and degree $-d$ gives a $T$ equivariant map $f_{\mathscr{S}}: \mathbb{P}^{1} \rightarrow 2_{d}$. When the family $\mathscr{S}$ is not trivial ( $T$-equivariantly isomorphic to a product $\mathscr{I}_{0} \times \mathbb{P}^{1}$ with $T$ acting trivially on the base $\left.\mathbb{P}^{1}\right)$, then its image is a $T$-invariant curve in $2_{d}$.

Here, we describe a collection of $T$-invariant curves whose tangent directions at each $T$-fixed point $(\boldsymbol{\delta}, \mathbf{a}, \mathbf{b})$ form a basis for $T_{(\boldsymbol{\delta}, \mathbf{a}, \mathbf{b})} 2_{d}$. We exhibit each curve as a subsheaf $\mathscr{Y}$ of $\mathbb{O}_{\mathbb{P}^{1} \times \mathbb{P}^{1}}^{n}$ of rank $n-r$ and degree $(-d,-1)$ with

$$
\mathscr{S}(1,0)=\mathscr{Y}_{(\boldsymbol{\delta}, \mathbf{a}, \mathbf{b})}, \quad \text { and } \quad \mathscr{S}(0,1)=\mathscr{Y}_{\left(\boldsymbol{\delta}^{\prime}, \mathbf{a}^{\prime}, \mathbf{b}^{\prime}\right)},
$$

where $\left(\boldsymbol{\delta}^{\prime}, \mathbf{a}^{\prime}, \mathbf{b}^{\prime}\right)$ is some other $T$-fixed point on $\mathscr{2}_{d}$. Each subsheaf $\mathscr{Y}$ is $T$ invariant, and so defines a $T$-invariant curve on $2_{d}$ connecting the two fixed points. Requiring that the second component of the degree is -1 guarantees that the family is not trivial.

We will show in the proof of Theorem 3.2 below that the differentials $d f_{\mathscr{S}}$ at the fixed points $[0,1],[1,0] \in \mathbb{P}^{1}$ are nonzero for each subsheaf $\mathscr{Y}$ that we consider. This implies that the maps $f_{\mathscr{S}}$ are closed immersions with image a smooth $T$ invariant curve. Further, we show that the tangent spaces to these curves form a basis of the tangent space at each $T$-fixed point which is compatible with the decomposition (2-1) (in fact, it coincides with the further decomposition of each summand of (2-1) into one-dimensional spaces spanned by monomials which was described following Theorem 2.1).

Each sheaf $\mathscr{Y}$ has one of three types: I, II, or III. We describe them below and then argue that they have the desired properties. We write $O$ for $O_{\mathbb{P}^{1} \times \mathbb{P}^{1}}$. We use the correspondence between sheaves over $\mathbb{O}$ and saturated modules over the bihomogeneous coordinate ring $k[x, y][s, t]$ of $\mathbb{P}_{[x, y]}^{1} \times \mathbb{P}_{[s, t]}^{1}$. Then $\mathbb{O}^{n}$ is the rank $n$ free module with basis $\mathbf{e}_{1}, \mathbf{e}_{2}, \ldots, \mathbf{e}_{n}$.

Type I: Let $\left(\boldsymbol{\delta}^{\prime}, \mathbf{a}^{\prime}, \mathbf{b}^{\prime}\right)$ be another fixed point where the data $(\boldsymbol{\delta}, \mathbf{a}, \mathbf{b})$ and $\left(\boldsymbol{\delta}, \mathbf{a}^{\prime}, \mathbf{b}^{\prime}\right)$ agree except in positions $i$ and $j$, with $\delta_{i}=\delta_{j}^{\prime}=1$ and $\delta_{j}=\delta_{i}^{\prime}=0$. Note that $a_{i}+b_{i}=a_{j}^{\prime}+b_{j}^{\prime}$. Let $\mathscr{Y}$ be the subsheaf of $\mathbb{O}^{n}$ which agrees with both $\mathscr{Y}_{(\boldsymbol{\delta}, \mathbf{a}, \mathbf{b})}$ and $\mathscr{Y}_{\left(\boldsymbol{\delta}^{\prime}, \mathbf{a}^{\prime}, \mathbf{b}^{\prime}\right)}$ except for its component in $\mathbb{O} \cdot \mathbf{e}_{i}+\mathcal{O} \cdot \mathbf{e}_{j}$, where it is the rank 1 and degree $-\left(a_{i}+b_{i}\right)$ subsheaf generated by the single element

$$
s x^{a_{i}} y^{b_{i}} \cdot \mathbf{e}_{i}+t x^{a_{j}^{\prime}} y^{b_{j}^{\prime}} \cdot \mathbf{e}_{j} .
$$

Type II: Let $\left(\boldsymbol{\delta}^{\prime}, \mathbf{a}^{\prime}, \mathbf{b}^{\prime}\right)$ be another $T$-fixed point where $\boldsymbol{\delta}=\boldsymbol{\delta}^{\prime}, \mathbf{b}=\mathbf{b}^{\prime}$, and the data $\mathbf{a}$ and $\mathbf{a}^{\prime}$ agree except in positions $i$ and $j$ with $i \neq j$. We suppose that $i$ and $j$ have been chosen so that $a_{i}<a_{i}^{\prime}$. Then $a_{j}>a_{j}^{\prime}$ and $c:=a_{i}^{\prime}-a_{i}=a_{j}-a_{j}^{\prime}>0$. Set

$$
\gamma:=a_{i}+b_{i}+c-a_{j}-b_{j}
$$


If $\gamma \geq 0$, let $\mathscr{S}$ be the subsheaf of $\mathscr{O}^{n}$ which agrees with both $\mathscr{Y}_{(\boldsymbol{\delta}, \mathbf{a}, \mathbf{b})}$ and $\mathscr{Y}_{\left(\boldsymbol{\delta}^{\prime}, \mathbf{a}^{\prime}, \mathbf{b}^{\prime}\right)}$ except for its components in $\mathbb{O} \cdot \mathbf{e}_{i}+\mathcal{O} \cdot \mathbf{e}_{j}$, where it is the subsheaf generated by

$$
x^{a_{i}+c} y^{b_{i}} \cdot \mathbf{e}_{i}, \quad x^{a_{j}} y^{b_{j}} \cdot \mathbf{e}_{j}, \quad \text { and } \quad s x^{a_{i}} y^{b_{i}} \cdot \mathbf{e}_{i}+t x^{a_{j}-c} y^{b_{j}+\gamma} \cdot \mathbf{e}_{j} .
$$

When $\gamma<0$, replace the third generator by $s x^{a_{i}} y^{b_{i}-\gamma} \cdot \mathbf{e}_{i}+t x^{a_{j}-c} y^{b_{j}} \cdot \mathbf{e}_{j}$.

The remaining sheaves of Type II are obtained by interchanging the roles of a and $\mathbf{b}$. That is, $\boldsymbol{\delta}=\boldsymbol{\delta}^{\prime}, \mathbf{a}=\mathbf{a}^{\prime}$ and $\mathbf{b}, \mathbf{b}^{\prime}$ agree except in positions $i \neq j$, and we further have that $c:=b_{i}^{\prime}-b_{i}=b_{j}-b_{j}^{\prime}>0$. Set

$$
\gamma:=a_{i}+b_{i}+c-a_{j}-b_{j} .
$$

If $\gamma \geq 0$, let $\mathscr{S}$ be as before, except with the generators (3-2) replaced by

$$
x^{a_{i}} y^{b_{i}+c} \cdot \mathbf{e}_{i}, \quad x^{a_{j}} y^{b_{j}} \cdot \mathbf{e}_{j}, \quad \text { and } s x^{a_{i}} y^{b_{i}} \cdot \mathbf{e}_{i}+t x^{a_{j}+\gamma} y^{b_{j}-c} \cdot \mathbf{e}_{j} .
$$

If $\gamma<0$, then the third generator will be $s x^{a_{i}-\gamma} y^{b_{i}} \cdot \mathbf{e}_{i}+t x^{a_{j}} y^{b_{j}-c} \mathbf{e}_{j}$.

Type III: Let $\left(\boldsymbol{\delta}^{\prime}, \mathbf{a}^{\prime}, \mathbf{b}^{\prime}\right)$ be another $T$-fixed point where $\boldsymbol{\delta}=\boldsymbol{\delta}^{\prime}$ and the data $(\mathbf{a}, \mathbf{b})$ and $\left(\mathbf{a}^{\prime}, \mathbf{b}^{\prime}\right)$ agree except in position $i$. Thus $\delta_{i} \neq 0$ and $a_{i}+b_{i}=a_{i}^{\prime}+b_{i}^{\prime}$. Let $\mathscr{S}$ be the subsheaf of $\mathscr{O}^{n}$ which agrees with both $\mathscr{S}_{(\boldsymbol{\delta}, \mathbf{a}, \mathbf{b})}$ and $\mathscr{S}_{\left(\boldsymbol{\delta}^{\prime}, \mathbf{a}^{\prime}, \mathbf{b}^{\prime}\right)}$, except for its component in $\mathcal{O} \cdot \mathbf{e}_{i}$, where it is the rank 1 and degree $-\left(a_{i}+b_{i}\right)$ subsheaf generated by

$$
s x^{a_{i}} y^{b_{i}} \cdot \mathbf{e}_{i}+t x^{a_{i}^{\prime}} y^{b_{i}^{\prime}} \cdot \mathbf{e}_{i} .
$$

Theorem 3.1. The subsheaves $\mathscr{Y}$ of $\mathrm{O}_{\mathbb{P}^{1} \times \mathbb{P}^{1}}^{n}$ of types I, II, and III are T-invariant and free of rank $n-r$ and degree $(-d,-1)$. They satisfy

$$
\mathscr{S}(1,0)=\mathscr{Y}_{(\boldsymbol{\delta}, \mathbf{a}, \mathbf{b})}, \quad \text { and } \quad \mathscr{Y}(0,1)=\mathscr{Y}_{\left(\boldsymbol{\delta}^{\prime}, \mathbf{a}^{\prime}, \mathbf{b}^{\prime}\right)},
$$

and hence define $T$-invariant curves on $2_{d}$.

Proof. The generators of $\mathscr{S}$ are $T$-invariant, except for those described by (3-1), (3-2), (3-3), and (3-4). But $T$ acts transitively on those generators for $s \cdot t \neq 0$. Therefore, each sheaf $\mathscr{Y}$ is $T$-invariant. In all cases, $\mathscr{Y}$ has degree -1 with respect to $\mathbb{P}_{[s, t]}^{1}$.

The theorem is clear for the sheaves of types I and III, as they are constant on $\mathbb{P}_{[s, t]}^{1}$, except for the rank 1 components (3-1) and (3-4), each of which has degree $\left(-\left(a_{i}+b_{i}\right),-1\right)$. Specializing these generators at $[s, t]=[1,0]$ and $[0,1]$ shows that $\mathscr{S}(1,0)=\mathscr{Y}_{(\boldsymbol{\delta}, \mathbf{a}, \mathbf{b})}$ and $\mathscr{S}(0,1)=\mathscr{Y}_{\left(\boldsymbol{\delta}^{\prime}, \mathbf{a}^{\prime}, \mathbf{b}^{\prime}\right)}$.

We use a Gröbner basis argument for the sheaves of type II. The Hilbert function for a submodule $M$ of $\mathrm{O}^{2}$ equals the Hilbert function for the module of leading terms of any Gröbner basis of $M$. As explained in [Eisenbud 1995, Chapter 15], a weight $\omega$ selecting these leading terms induces a $\mathbb{G}_{m}$-action on $\mathbb{O}^{2}$ whose restriction 
to the Gröbner basis of $M$ generates a flat family over $\mathbb{A}^{1}$ whose special fibre is the module of leading terms.

For now, set $s=t=1$. If $\mathbf{e}_{i}>\mathbf{e}_{j}$, then the generators (3-2) form a Gröbner basis for any position-over-monomial ordering, and the third generator has leading term $x^{a_{i}} y^{b_{i}} \cdot \mathbf{e}_{i}$. As $c>0$, the module of leading terms is generated by $x^{a_{i}} y^{b_{i}} \cdot \mathbf{e}_{i}$ and $x^{a_{j}} y^{b_{j}} \cdot \mathbf{e}_{j}$, and so it has rank 2 and degree $-\left(a_{i}+b_{i}+a_{j}+b_{j}\right)$. The weight $\omega$ with $\omega\left(\mathbf{e}_{i}\right)=0$ and $\omega\left(\mathbf{e}_{j}\right)=-1$ induces the leading terms and has corresponding $\mathbb{G}_{m}$-action $t \cdot\left(\mathbf{e}_{i}, \mathbf{e}_{j}\right)=\left(\mathbf{e}_{i}, t \mathbf{e}_{j}\right)$, for $t \in \mathbb{G}_{m}$. This action on $\mathscr{S}(1,1)$ is the flat family of modules over $\mathbb{A}^{1}$ generated by

$$
x^{a_{i}+c} y^{b_{i}} \cdot \mathbf{e}_{i}, \quad x^{a_{j}} y^{b_{j}} \cdot \mathbf{e}_{j}, \quad \text { and } \quad x^{a_{i}} y^{b_{i}} \cdot \mathbf{e}_{i}+t x^{a_{j}-c} y^{b_{j}+\gamma} \cdot \mathbf{e}_{j},
$$

which is just the part of $\mathscr{Y}$ in $\mathcal{O} \cdot \mathbf{e}_{i}+\mathcal{O} \cdot \mathbf{e}_{j}$ restricted to the affine subset $U$ of $\mathbb{P}_{[s, t]}^{1}$ where $s \neq 0$. Thus $\left.\mathscr{S}\right|_{U}$ is a flat family over $U$ of free subsheaves of $O_{\mathbb{P} 1}^{n}$ of rank $n-r$ and degree $-d$, and $\mathscr{Y}(1,0)=\mathscr{Y}_{(\boldsymbol{\delta}, \mathbf{a}, \mathbf{b})}$.

When $s=t=1$ the generators (3-2) form a Gröbner basis when $\mathbf{e}_{i}<\mathbf{e}_{j}$, where the third generator has leading term $x^{a_{j}-c} y^{b_{j}+\gamma} \cdot \mathbf{e}_{j}$. The module of leading terms is generated by

$$
x^{a_{i}+c} y^{b_{i}} \cdot \mathbf{e}_{i}, \quad x^{a_{j}} y^{b_{j}} \cdot \mathbf{e}_{j}, \quad \text { and } \quad x^{a_{j}-c} y^{b_{j}+\gamma} \cdot \mathbf{e}_{j} .
$$

Since $\gamma \geq 0$ and $c>0$, saturating the ideal of $k[x, y]$ generated by $x^{a_{j}} y^{b_{j}}$ and $x^{a_{j}-c} y^{b_{j}+\gamma}$ by the irrelevant maximal ideal generated by $x$ and $y$ gives the ideal generated by $x^{a_{j}-c} y^{b_{j}}$. Thus the module of leading terms is generated by

$$
x^{a_{i}+c} y^{b_{i}} \cdot \mathbf{e}_{i}, \quad \text { and } \quad x^{a_{j}-c} y^{b_{j}} \cdot \mathbf{e}_{j} .
$$

As before, restricting $\mathscr{Y}$ to the affine set of points $[s, t]$ of $\mathbb{P}^{1}$ where $t \neq 0$ gives a flat family of subsheaves of $\mathbb{O}_{\mathbb{P} 1}^{n}$ of rank $n-r$ and degree $-d$ with special fibre $\mathscr{S}(0,1)=\mathscr{Y}_{\left(\boldsymbol{\delta}^{\prime}, \mathbf{a}^{\prime}, \mathbf{b}^{\prime}\right)}$. The same arguments suffice for the module generated by (3-3).

Theorem 3.2. All of the T-invariant curves induced by the sheaves 9 of types $\mathrm{I}$, II, and III are smooth. For any $T$-fixed point $\mathscr{Y}_{(\boldsymbol{\delta}, \mathbf{a}, \mathbf{b})}$ in $2_{d}$, the set of tangent directions to the curves which contain this point corresponds to the T-basis of $T_{(\boldsymbol{\delta}, \mathbf{a}, \mathbf{b})} 2_{d}$ defined in Section 2, and this correspondence is $T$-equivariant, respecting the weights. More specifically, at the $T$-fixed point $\mathscr{S}_{(\boldsymbol{\delta}, \mathbf{a}, \mathbf{b})}$,

I. The weight of Type I curve (3-1) is

$$
\mathbf{e}_{j}-\mathbf{e}_{i}+\left(a_{j}^{\prime}-a_{i}\right) \mathbf{f}
$$

and such curves correspond to the first summand of (2-1). 
II. The weight of Type II curve (3-2) is

$$
\mathbf{e}_{j}-\mathbf{e}_{i}+\left(a_{j}^{\prime}-a_{i}\right) \mathbf{f},
$$

the weight of Type II curve (3-3) is

$$
\mathbf{e}_{j}-\mathbf{e}_{i}+\left(b_{i}-b_{j}^{\prime}\right) \mathbf{f},
$$

and such curves correspond to the second summand of (2-1) when $i \neq j$.

III. The weight of Type III curve (3-4) is

$$
\left(a_{j}^{\prime}-a_{i}\right) \mathbf{f},
$$

and such curves correspond to the second summand of (2-1) when $i=j$.

Proof. In what follows, we work locally near $[1,0]$ by setting $s=1$. The same arguments handle the other fixed point $[0,1]$.

Note that the generator (3-1) of a Type I sheaf may be rewritten

$$
x^{a_{i}} y^{b_{i}} \cdot\left(\mathbf{e}_{i}+t \cdot x^{a_{j}^{\prime}-a_{i}} y^{b_{j}^{\prime}-b_{i}} \mathbf{E}_{i j}\left(\mathbf{e}_{i}\right)\right) .
$$

This shows that the differential $d f_{\varphi}$ at $[1,0]$ maps onto the span of the $T$-basis element $x^{a_{j}^{\prime}-a_{i}} y^{b_{j}^{\prime}-b_{i}} \mathbf{E}_{i j}$ of $\operatorname{Hom}\left(\mathscr{S}_{a_{i}, b_{i}} \cdot \mathbf{e}_{i}, \mathcal{O}_{\mathbb{P} 1} \cdot \mathbf{e}_{j}\right)$. Thus the Type I curves are smooth, and their tangent spaces at $\mathscr{S}_{(\boldsymbol{\delta}, \mathbf{a}, \mathbf{b})}$ span the component of $T_{(\boldsymbol{\delta}, \mathbf{a}, \mathbf{b})} \mathscr{2}_{d}$ given by the first summand of (2-1). (Recall that in Type I, we have $\delta_{i}=\delta_{j}^{\prime}=1$ and $\delta_{j}=\delta_{i}^{\prime}=0$. .)

A similar analysis shows that the tangent space at $t=0$ of the Type III curve defined by (3-4) is spanned by $x^{a_{i}^{\prime}-a_{i}} y^{b_{i}^{\prime}-b_{i}} \mathbf{E}_{i i}$, and so the tangent spaces at $\mathscr{Y}_{(\boldsymbol{\delta}, \mathbf{a}, \mathbf{b})}$ of Type III curves span the component of $T_{(\boldsymbol{\delta}, \mathbf{a}, \mathbf{b})} 2_{d}$ given by the second summand of (2-1) when $i=j$.

For a curve of type II, note that the family of sheaves described by (3-2) is constant in a neighborhood of $\infty$. In a neighborhood of 0 , it is given by

$$
x^{a_{i}+c} \mathbf{e}_{i}, \quad x^{a_{j}} \mathbf{e}_{j}, \quad \text { and } \quad x^{a_{i}} \cdot\left(\mathbf{e}_{i}+t x^{\left(a_{j}-c\right)-a_{i}} \mathbf{E}_{i j}\left(\mathbf{e}_{i}\right)\right) .
$$

Thus $x^{\left(a_{j}-c\right)-a_{i}} \mathbf{E}_{i j}$ spans the tangent space at $t=0$. A similar argument near $\infty$ for the sheaves described by (3-3) shows that the tangent spaces of Type II curves at $\mathscr{Y}_{(\boldsymbol{\delta}, \mathbf{a}, \mathbf{b})}$ span the component of $T_{(\boldsymbol{\delta}, \mathbf{a}, \mathbf{b})} 2_{d}$ given by the second summand of (2-1) when $i \neq j$.

A moment graph of a $T$-variety is a graph whose vertices correspond to $T$ fixed points and whose edges correspond to $T$-invariant curves, embedded into $\mathbb{R} \otimes \operatorname{Hom}(T, \mathbb{Z})$ so that the edge corresponding to a $T$-invariant curve is parallel to the weight of the action of $T$ on the curve. More specifically, if $C$ is a $T$-invariant curve joining fixed points $p$ and $q$, then the edge from $p$ to $q$ in the moment graph is a positive multiple of the $T$-weight of $T_{p} C$. When $k=\mathbb{C}$ and we fix a Kähler 
form, there is a moment map $\mu: 2_{d} \rightarrow \mathfrak{t}^{*}$ and the image of the $T$-fixed points and $T$-invariant curves is a moment graph.

When there are finitely many $T$-invariant curves, the Goresky-Kottwitz-MacPherson method to compute equivariant cohomology is conveniently expressed in terms of a moment graph, with one relation for each edge. When there are infinitely many $T$-invariant curves, there are additional relations coming from families of $T$-invariant curves, so it is better to work with the moment multigraph, where each family of $T$-invariant curves (which will appear as a connected component of parallel edges in the moment graph) is considered to form a single multiedge with more than 2 vertices, given by the fixed points in the closure of the family. To have a structure which determines the equivariant cohomology or Chow groups, we should label each multiedge with the topological type of the corresponding family.

Guillemin and Zara [2001; 2002; 2003] have explored the combinatorial properties of moment graphs.

Example 3.3. Figure 1 represents a moment multigraph of $\mathscr{2}_{2}(0,2)$. Since the $T$-fixed points

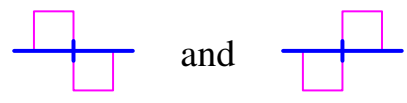

have the same image in this and in any moment multigraph, we displace their images from their true positions for clarity. Similarly, some images of $T$-invariant curves are displaced or drawn as arcs.

The $T$-basis of the tangent space at

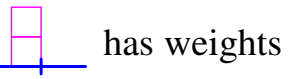

$$
\left\{\mathbf{e}_{1}-\mathbf{e}_{2}, \mathbf{e}_{1}-\mathbf{e}_{2}+\mathbf{f},-\mathbf{f},-2 \mathbf{f}\right\}
$$

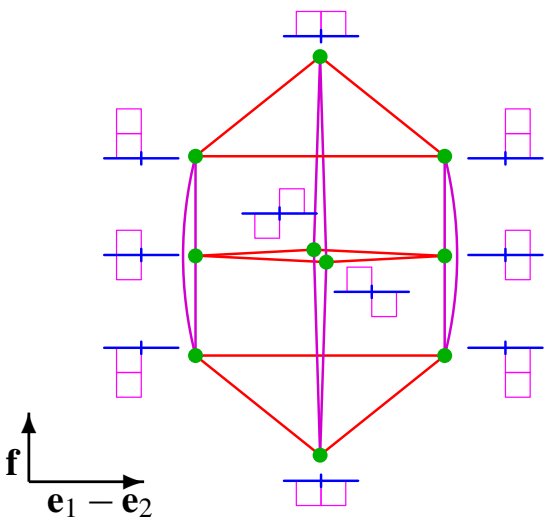

Figure 1. A moment multigraph of $2_{2}(0,2)$. 
These correspond to the following four $T$-invariant curves:

\begin{tabular}{l|cccc} 
Generators of submodules of $\mathcal{O}^{2}$ & $t=0$ & $s=0$ & Weight & Type \\
\hline$x^{2} \mathbf{e}_{1}, x^{2} \mathbf{e}_{2}, t \mathbf{e}_{1}+s \mathbf{e}_{2}$ & $x^{2} \mathbf{e}_{1}, 1 \cdot \mathbf{e}_{2}$ & $\mathbf{e}_{1}, x^{2} \mathbf{e}_{2}$ & $1 \cdot \mathbf{e}_{1}-\mathbf{e}_{2}$ & II \\
$x^{2} \mathbf{e}_{1}, x^{2} \mathbf{e}_{2}, t x \mathbf{e}_{1}+s y \mathbf{e}_{2}$ & $x^{2} \mathbf{e}_{1}, 1 \cdot \mathbf{e}_{2}$ & $x \mathbf{e}_{1}, x \mathbf{e}_{2}$ & $\mathbf{e}_{1}-\mathbf{e}_{2}+\mathbf{f}$ & II \\
$\left(s x^{2}+t x y\right) \mathbf{e}_{1}, \mathbf{e}_{2}$ & $x^{2} \mathbf{e}_{1}, 1 \cdot \mathbf{e}_{2}$ & $x y \mathbf{e}_{1}, 1 \cdot \mathbf{e}_{2}$ & $-\mathbf{f}$ & III \\
$\left(s x^{2}+t y^{2}\right) \mathbf{e}_{1}, \mathbf{e}_{2}$ & $x^{2} \mathbf{e}_{1}, 1 \cdot \mathbf{e}_{2}$ & $y^{2} \mathbf{e}_{1}, 1 \cdot \mathbf{e}_{2}$ & $-2 \mathbf{f}$ & III
\end{tabular}

The $T$-basis to the tangent space at $\square$ has weights

$$
\left\{ \pm \mathbf{f}, \pm\left(\mathbf{e}_{2}-\mathbf{e}_{1}\right)\right\} .
$$

These correspond to the following four $T$-invariant curves:

\begin{tabular}{l|cccc} 
Generators of submodules of $\mathcal{O}^{2}$ & $t=0$ & $s=0$ & Weight & Type \\
\hline$(s x+t y) \mathbf{e}_{1}, y \mathbf{e}_{2}$ & $x \mathbf{e}_{1}, y \mathbf{e}_{2}$ & $y \mathbf{e}_{1}, y \mathbf{e}_{2}$ & $-\mathbf{f}$ & III \\
$x y \mathbf{e}_{1}, y \mathbf{e}_{2}, s x \mathbf{e}_{1}+t x \mathbf{e}_{2}$ & $x \mathbf{e}_{1}, y \mathbf{e}_{2}$ & $x y \mathbf{e}_{1}, \mathbf{e}_{2}$ & $\mathbf{e}_{2}-\mathbf{e}_{1}$ & II \\
$x \mathbf{e}_{1}, x y \mathbf{e}_{2}, t y \mathbf{e}_{1}+s y \mathbf{e}_{2}$ & $x \mathbf{e}_{1}, y \mathbf{e}_{2}$ & $\mathbf{e}_{1}, x y \mathbf{e}_{2}$ & $\mathbf{e}_{1}-\mathbf{e}_{2}$ & II \\
$x \mathbf{e}_{1},(s y+t x) \mathbf{e}_{2}$ & $x \mathbf{e}_{1}, y \mathbf{e}_{2}$ & $x \mathbf{e}_{1}, x \mathbf{e}_{2}$ & $\mathbf{f}$ & III
\end{tabular}

\section{Families of $T$-invariant curves}

Suppose that $Z$ is a $T$-invariant curve on $2_{d}$. Let $T^{\prime}$ be the identity component of the stabilizer in $T$ of a general point of $Z$. It is a codimension one subtorus of $T$, so we can choose an isomorphism $T / T^{\prime} \simeq \mathbb{G}_{m}$. The action of $T$ on $Z$ factors through the resulting quotient

$$
T \longrightarrow T / T^{\prime} \simeq \mathbb{G}_{m} .
$$

This composition $\eta: T \rightarrow \mathbb{G}_{m}$ is a primitive weight. When $T$ is smooth, it is a multiple of the weight of the action of $T$ on the tangent space to $Z$ at either fixed point.

Let $Y$ be the component of the $T^{\prime}$-fixed point locus $2_{d}^{T^{\prime}}$ which contains $Z$; since $2_{d}$ is smooth, so is $Y$. Then $Y \backslash Y^{T}$ is foliated by one-dimensional orbits of $T$ whose closures are $T$-invariant curves. We call $Y$ the family of $T$-invariant curves on $2_{d}$ which contains $Z$. If

$$
p \in Y^{T} \subset 2_{d}^{T}
$$

is a $T$-fixed point of $Y$, then $T_{p} Y$ is a $T$-invariant linear subspace of $T_{p} 2_{d}$ which is fixed pointwise by $T^{\prime}$. In particular, all weights of the $T$-action on $T_{p} Y$ are multiples of $\eta$.

To classify families of $T$-invariant curves, we first determine which $T$-weights of $T_{p} 2_{d}$ are parallel, using Theorem 3.2. If the weight of a curve is not parallel to the weight of any other curve, the curve is isolated. 
Theorem 4.1. Two $T$-invariant curves $\mathscr{Y}, \mathscr{S}^{\prime}$ of types I, II, or III containing the fixed point $\mathscr{S}_{(\boldsymbol{\delta}, \mathbf{a}, \mathbf{b})}$ have parallel $T$-weights if and only if either

(1) both $\mathscr{Y}$ and $\mathscr{S}^{\prime}$ have Type III or

(2) both $\mathscr{Y}$ and $\mathscr{Y}^{\prime}$ have Type II, and

(a) $\mathscr{Y}$ connects $\mathscr{Y}_{(\boldsymbol{\delta}, \mathbf{a}, \mathbf{b})}$ to $\mathscr{Y}_{\left(\boldsymbol{\delta}, \mathbf{a}^{\prime}, \mathbf{b}\right)}$,

(b) $\mathscr{S}^{\prime}$ connects $\mathscr{Y}_{(\boldsymbol{\delta}, \mathbf{a}, \mathbf{b})}$ to $\mathscr{Y}_{\left(\boldsymbol{\delta}, \mathbf{a}, \mathbf{b}^{\prime}\right)}$, and

(c) $\mathbf{a}$ and $\mathbf{a}^{\prime}$ agree except in positions $i$ and $j$ with $i \neq j, \mathbf{b}$ and $\mathbf{b}^{\prime}$ agree except in positions $i$ and $j$ (same $i, j$ ), $a_{i}+b_{i}=a_{j}^{\prime}+b_{j}^{\prime}$.

Proof. For (1), note that the weight of $T$ on a Type III curve is parallel to $\mathbf{f}$.

If a curve does not have type III, then its weight has the form

$$
\mathbf{e}_{j}-\mathbf{e}_{i}+c \mathbf{f},
$$

where $\delta_{i}=1$ and either $\delta_{j}=0$ if it has type I or $\delta_{j}=1$ if it has type II. Thus $\mathscr{Y}$ and $\mathscr{S}^{\prime}$ have the same weight and type, $\delta=\delta^{\prime}$, and the indices $i$ and $j$ in their definitions coincide.

Weights of curves of types I and II correspond to (2-2) and (2-3), respectively. Inspecting (2-2) shows that no two curves of type I can have the same weight. Inspecting (2-3) reveals that either a given curve $\mathscr{Y}$ of type II has a unique weight, or else there is exactly one other Type II curve $\mathscr{S}^{\prime}$ with the same weight, and the two curves are as described in the statement of the theorem.

We show that all Type III curves at a fixed point lie in a single family of $T$ invariant curves, and if two Type II curves have the same weight then they lie in a 2-dimensional family. Together with the isolated $T$-invariant curves, this shows that the tangent spaces at a given fixed point $p$ to families of $T$-invariant curves are the subspaces of $T_{p} 2_{d}$ which are stabilized by codimension 1 subtori of $T$. It follows that these families contain all $T$-invariant curves in $2_{d}$.

To see this, fix a weight and consider the family $Y$ of all $T$-invariant curves meeting $p$ that have that weight or a parallel weight. The common kernel of these parallel weights is a codimension 1 subtorus $T^{\prime}$ of $T$, which stabilises this family pointwise. Since $2_{d}$ is smooth, $Y$ is smooth, and its tangent space at $p$ is necessarily the $T^{\prime}$-fixed subspace of the tangent space of $2_{d}$ at $p$. In particular the dimension of $Y$ is the dimension of this $T^{\prime}$-fixed subspace. Since the families described in Theorem 4.1 whose weights annihilate $T^{\prime}$ have dimension equal to the dimension of the $T^{\prime}$-fixed subspace, there can be no other $T^{\prime}$-stable curves.

Vertical families. The vertical family containing $\mathscr{S}_{(\boldsymbol{\delta}, \mathbf{a}, \mathbf{b})}$ is parametrized by the product of projective spaces

$$
\prod_{\delta_{i}=1} \mathbb{P} H^{0}\left(\mathbb{O}\left(a_{i}+b_{i}\right)\right) \simeq \prod_{\delta_{i}=1} \mathbb{P}^{a_{i}+b_{i}} .
$$


It contains exactly the fixed points $\mathscr{Y}_{\left(\boldsymbol{\delta}^{\prime}, \mathbf{a}^{\prime}, \mathbf{b}^{\prime}\right)}$ where $\boldsymbol{\delta}=\boldsymbol{\delta}^{\prime}$, and $\mathbf{a}^{\prime}+\mathbf{b}^{\prime}=\mathbf{a}+\mathbf{b}$, along with all Type III curves which connect them. This includes all Type III curves at each of these fixed points.

Consider the family $\mathscr{S}$ of submodules of $\mathrm{O}_{\mathbb{P}^{1}}^{n}$ generated by

$$
\left\{\mathbf{e}_{i} s_{i} \mid \delta_{i}=1, s_{i} \in H^{0}\left(\mathcal{O}\left(a_{i}+b_{i}\right)\right)\right\} .
$$

The base of this family is

$$
\prod_{\delta_{i}=1} \mathbb{P} H^{0}\left(\mathcal{O}\left(a_{i}+b_{i}\right)\right)
$$

all subsheaves have rank $n-r$ and degree $-d$, and the foliation by $T$-invariant curves is given by the $T$-action on the base.

Horizontal families. If there exist $i, j, c, c^{\prime}$ such that $1 \leq c \leq a_{j}, 1 \leq c^{\prime} \leq b_{j}$, and $a_{i}+b_{i}+c+c^{\prime}=a_{j}+b_{j}$, then the point $\mathscr{S}_{(\boldsymbol{\delta}, \mathbf{a}, \mathbf{b})}$ lies in a horizontal family parametrized by the product of two projective lines. Let

$$
a_{i}^{\prime}=a_{i}+c, a_{j}^{\prime}=a_{j}-c, b_{i}^{\prime}=b_{i}+c^{\prime}, b_{j}^{\prime}=b_{j}-c^{\prime} .
$$

Let $([s, t],[\sigma, \tau])$ be the coordinates of $\mathbb{P}^{1} \times \mathbb{P}^{1}$, and let $\mathscr{Y}$ be the submodule of $\mathcal{O}_{\mathbb{P} 1}^{n}$ which, except for its components in $\mathcal{O} \cdot \mathbf{e}_{i}+\mathcal{O} \cdot \mathbf{e}_{j}$, agrees with $\mathscr{Y}_{(\boldsymbol{\delta}, \mathbf{a}, \mathbf{b})}$. The component of $\mathscr{Y}$ in $\mathcal{O} \cdot \mathbf{e}_{i}+\mathscr{O} \cdot \mathbf{e}_{j}$ is the subsheaf generated by

$$
\mathbf{e}_{j} x^{a_{j}} y^{b_{j}}, s \mathbf{e}_{i} x^{a_{i}^{\prime}} y^{b_{i}}+t \mathbf{e}_{j} x^{a_{j}} y^{b_{j}^{\prime}}, \sigma \mathbf{e}_{i} x^{a_{i}} y^{b_{i}^{\prime}}+\tau \mathbf{e}_{j} x^{a_{j}^{\prime}} y^{b_{j}}, \text { and } \mathbf{e}_{i} x^{a_{i}^{\prime}} y^{b_{i}^{\prime}} .
$$

Similar reasoning as for Theorem 3.1 shows that this defines a family of $T$-invariant curves over the base $\mathbb{P}^{1} \times \mathbb{P}^{1}$ with coordinates $([s, t],[\sigma, \tau])$. It contains four $T$ fixed points: setting $t=\tau=0$ gives the fixed point $\mathscr{S}_{(\boldsymbol{\delta}, \mathbf{a}, \mathbf{b})}$, setting $t=\sigma=0$ gives the fixed point $\mathscr{S}_{\left(\boldsymbol{\delta}, \mathbf{a}^{\prime}, \mathbf{b}\right)}$, setting $s=\tau=0$ gives the fixed point $\mathscr{Y}_{\left(\boldsymbol{\delta}, \mathbf{a}, \mathbf{b}^{\prime}\right)}$, and setting

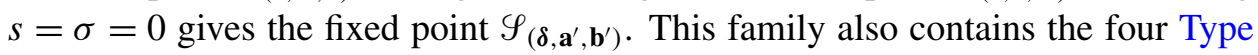
II curves connecting these four fixed points, given by setting exactly one of $s, t, \sigma$, or $\tau$ equal to zero. Furthermore, the data $(\boldsymbol{\delta}, \mathbf{a}, \mathbf{b})$ and $\left(\boldsymbol{\delta}^{\prime}, \mathbf{a}^{\prime}, \mathbf{b}^{\prime}\right)$ satisfy Theorem 4.1(2)(c), and any two $T$-invariant curves $\mathscr{S}$ and $\mathscr{S}^{\prime}$ as in Theorem 4.1(2) lie in a unique horizontal family.

We summarize the results of this section, in which we identified the fixed point loci of codimension 1 subtori of $T$.

Theorem 4.2. The connected components of the fixed point loci of codimension 1 subtori of $T$ which contain the fixed point $\mathscr{Y}_{(\boldsymbol{\delta}, \mathbf{a}, \mathbf{b})}$ are

(1) Type I curves, which are isolated;

(2) Type II curves whose weight at $\mathscr{Y}_{(\boldsymbol{\delta}, \mathbf{a}, \mathbf{b})}$ is unique, which are also isolated; 
(3) horizontal families, which occur only when there are two curves of type II containing $\mathscr{Y}_{(\boldsymbol{\delta}, \mathbf{a}, \mathbf{b})}$ with the same weight (these are isomorphic to $\mathbb{P}^{1} \times \mathbb{P}^{1}$ and contain these two Type II curves);

(4) one vertical family, which is isomorphic to a product of $n-r$ projective spaces and has dimension equal to $d$ (all curves of type III which contain $\mathscr{S}_{(\delta, \mathbf{a}, \mathbf{b})}$ lie in this family).

\section{Algebraic extension of GKM theory}

We discuss equivariant localization and an extension of the Goresky-KottwitzMacPherson relations when there are finitely many fixed points but infinitely many $T$-invariant curves.

We work with equivariant Chow rings; similar results hold for equivariant cohomology. In fact, when $k=\mathbb{C}, X$ is smooth and projective, and $X^{T}$ is finite, the two theories coincide.

We first recall some properties of $T$-equivariant Chow rings as developed by Edidin and Graham [1998a] and Brion [1997]. Next, we outline Evain's [2007] development of ideas of Brion which extends the GKM relations to describe the $T$-equivariant Chow ring of a smooth variety with finitely many $T$-fixed points when there are infinitely many $T$-invariant curves. This description involves idealmembership relations, one for each generator of the equivariant Chow ring of each family of $T$-invariant curves. When the generators are given by smooth subvarieties, these relations may be expressed in terms of tangent weights. This gives one form of our presentation for $A_{T}^{*}\left(2_{d}\right)$ in Theorem 1.1.

We next give a variant of these relations using differential operators, which gives the other form of our presentation for $A_{T}^{*}\left(2_{d}\right)$.

We then compute these relations for products of projective spaces, and finally deduce Theorem 1.1.

5A. Torus equivariant Chow rings. When a linear algebraic group $G$ acts on a smooth scheme $X$, Edidin and Graham [1998a] defined the equivariant Chow ring $A_{G}^{*}(X)$, using Totaro's algebraic approximation to the classifying space of $G$. It satisfies functorial properties under equivariant maps analogous to those for ordinary Chow rings [Fulton 1998], including proper pushforwards and pullbacks by local complete intersection morphisms.

When the group is a torus $T$, Brion [1997] gave an alternative development of this theory which includes versions of the localization theorems that hold for equivariant cohomology. He gave the following presentation for the equivariant Chow ring, analogous to the usual presentation of Chow groups. The equivariant Chow ring $A_{T}^{*}(p)$ of a point $p$ is the integral symmetric algebra $S$ of the character group $\hat{T}$ of $T$. Equivariant pullback makes $A_{T}^{*}(X)$ into an $S$-module. 
Proposition 5.1 [Brion 1997, Theorem 2.1]. The $S$-module $A_{T}^{*}(X)$ is defined by generators $[Y]$, for each $T$-invariant subvariety $Y$, and by relations

$$
\left[\operatorname{div}_{Y}(f)\right]-\chi[Y]
$$

for each rational function $f$ on $Y$ which is a T-eigenvector of weight $\chi$; here $\chi$ is considered as an element of $S$ in degree 1.

The usual Chow ring may be recovered from the $S$-module $A_{T}^{*}(X)$, as the quotient by the ideal $S^{+}$of $S$ generated by the character group $\hat{T}$.

Proposition 5.2 [Brion 1997, Corollary 2.3.1].

$$
A^{*}(X)=A_{T}^{*}(X) \otimes_{S} \mathbb{Z}=A_{T}^{*}(X) / S^{+} A_{T}^{*}(X) .
$$

The analogous statement in equivariant cohomology requires stronger hypotheses.

When $k=\mathbb{C}$, the connection between Chow groups and cohomology is given by the cycle map

$$
A_{T}^{*}(X) \rightarrow H_{T, c}^{*}(X, \mathbb{Z})
$$

to compactly supported (Borel-Moore) equivariant cohomology. If $X$ is projective and the fixed point set $X^{T}$ is finite, then the cycle map is an isomorphism.

Some statements below hold only for the rational equivariant Chow ring

$$
A_{T}^{*}(X)_{\mathbb{Q}}:=A_{T}^{*}(X) \otimes_{\mathbb{Z}} \mathbb{Q} .
$$

This is a module over the rational equivariant Chow ring $A_{T}^{*}(p)_{\mathbb{Q}}$ of a point $p$, which is the symmetric algebra $S_{\mathbb{Q}}$ of $\hat{T}_{\mathbb{Q}}:=\hat{T} \otimes_{\mathbb{Z}} \mathbb{Q}$.

5B. Localization. We now assume that $X^{T}$ is finite, and that $X$ has a decomposition into $T$-invariant affine cells $C_{1}, \ldots, C_{m}$ which can be ordered so that for $i=1, \ldots, m$, the union $C_{1} \cup \cdots \cup C_{i}$ is Zariski open. We will call such varieties filtrable; this is close to the terminology of Brion [1997], but he did not require $X^{T}$ to be finite, and his cells were allowed to be vector bundles over components of $X^{T}$. If $X$ is smooth and projective and $X^{T}$ is finite, then Białynicki-Birula [1973] showed that it is filtrable.

Let $i: X^{T} \rightarrow X$ be the inclusion of the subscheme of $T$-fixed points of $X$.

Proposition 5.3 [Brion 1997, Corollary 3.2.1]. The S-module $A_{T}^{*}(X)$ is free. The map

$$
i^{*}: A_{T}^{*}(X) \rightarrow A_{T}^{*}\left(X^{T}\right)
$$

is an injection.

Brion also established Chow ring versions of the results of Chang and Skjelbred and of Goresky, Kottwitz, and MacPherson concerning the image of the localization map. 
Proposition 5.4 [Brion 1997, Sections 3.3 and 3.4]. (a) The image of the localization map $i^{*}: A_{T}^{*}(X)_{\mathbb{Q}} \rightarrow A_{T}^{*}\left(X^{T}\right)_{\mathbb{Q}}$ is the intersection of the images of the localization maps

$$
i_{T^{\prime}}^{*}: A_{T}^{*}\left(X^{T^{\prime}}\right)_{\mathbb{Q}} \rightarrow A_{T}^{*}\left(X^{T}\right)_{\mathbb{Q}},
$$

where $T^{\prime}$ runs over all codimension one subtori of $T$.

(b) When $T$ acts with finitely many fixed points and has finitely many invariant curves, then the image of the localization map

$$
i^{*}: A_{T}^{*}(X)_{\mathbb{Q}} \rightarrow A_{T}^{*}\left(X^{T}\right)_{\mathbb{Q}} \simeq\left(S_{\mathbb{Q}}\right)^{X^{T}}
$$

is the set of all tuples $\left(f_{p}\right)_{p \in X^{T}} \in\left(S_{\mathbb{Q}}\right)^{X^{T}}$ such that whenever $p$ and $q$ belong to the same irreducible $T$-invariant curve $C$, we have $f_{p} \equiv f_{q}$ modulo $\chi$, where $\chi$ is the weight of the action of $T$ on $T_{p} C$.

Statement (a) is analogous to a theorem of Chang and Skjelbred [1974] for equivariant cohomology. This result, together with the easy calculation of the equivariant Chow groups of $\mathbb{P}^{1}$, immediately gives (b), which is the Chow analog of the GKM relations for equivariant cohomology.

In general this result does not hold with $\mathbb{Z}$ coefficients. For instance, suppose that $\operatorname{dim} X=2, x \in X^{T}$, and the weights of $T$ on the tangent space $T_{x} X$ are $a \chi$ and $a^{\prime} \chi^{\prime}$, where $\chi, \chi^{\prime} \in \hat{T}$ are linearly independent primitive characters. Then condition (b) would say that if $\left(f_{p}\right) \in A_{T}^{*}\left(X^{T}\right)$ has $f_{p}=0$ for $p \neq x$, then it is in the image of $i^{*}$ if $f_{x}$ is a multiple of $\operatorname{lcm}\left(a, a^{\prime}\right) \chi \chi^{\prime}$. In fact, $f_{x}$ must be a multiple of $a a^{\prime} \chi \chi^{\prime}$.

This is essentially the only obstruction to working with $\mathbb{Z}$ coefficients, at least if the fixed point set is finite. We say that the tangent weights of a $T$-variety $X$ are almost coprime if whenever two $T$-weights of $T_{p} X$ for $p \in X^{T}$ are divisible by the same integer $a>1$, then they are parallel. With this added hypothesis, Brion's proof of Proposition 5.4 works over $\mathbb{Z}$.

Theorem 5.5. Let $X$ be a smooth filtrable $T$-variety whose tangent weights are almost coprime. Then Proposition 5.4 holds with rational Chow groups replaced by integral Chow groups.

5C. Evain's relations. When $T$ does not have finitely many invariant curves on $X$, then statement (b) of Proposition 5.4 fails, but by (a) we can still compute $A_{T}^{*}(X)$ if we know the images of $i_{T^{\prime}}^{*}$ for all codimension one subtori $T^{\prime}$ of $T$. A finite set of such $T^{\prime}$ suffices, namely those which fix at least one $T$-invariant curve pointwise. Brion [1997] and Goldin and Holm [2001] have computed cases where the components of $X^{T^{\prime}}$ are low-dimensional. Evain [2007] recently described relations in the general case: 
Let $Y=X^{T^{\prime}}$ for $T^{\prime}$ a codimension one subtorus of $T$. By [Iversen 1972], $Y$ is smooth. For $p \in Y^{T}=X^{T}$, let

$$
e_{p}^{T}(Y)=\left.e^{T}(T Y)\right|_{p}
$$

be the localization of the equivariant Euler class of $T Y$ at $p$. Under the identification $A_{T}^{*}(p)=S$, this is the product of the $T$-weights on the tangent space $T_{p} Y$.

Proposition 5.6 [Evain 2007, Corollary 27]. A class $\alpha=\left(\alpha_{p}\right)_{p \in Y^{T}}$ in $S^{Y^{T}}$ lies in $i_{T^{\prime}}^{*} A_{T}^{*}(Y)$ if and only if

$$
\sum_{p \in Y^{T}} \frac{\alpha_{p} \beta_{p}}{e_{p}^{T}(Y)} \in S
$$

for every $\beta \in i_{T^{\prime}}^{*} A_{T}^{*}(Y)$.

Remark (On Evain's proof). The condition (5-1) is necessary, since if $\pi$ is the projection of $Y$ to a point, then the sum is simply $\pi_{*}(\alpha \cdot \beta)$, by the integration formula of Edidin and Graham [1998b]. Note that since $\pi_{*}$ is $S$-linear, it is enough to take $\beta$ in a generating set of the $S$-module $i^{*} A_{T}^{*}(Y)$.

By [Białynicki-Birula 1973], there are two $T$-invariant cell decompositions $C_{p}^{+}$ and $C_{p}^{-}$for $p \in Y^{T}$ of $Y$ and an ordering of the fixed points $Y^{T}$ such that the matrix with entries in $S$ whose $(p, q)$-entry is

$$
\pi_{*}\left(\left[C_{p}^{+}\right] \cdot\left[C_{q}^{-}\right]\right)
$$

is unitriangular. Either set of classes $\left[C_{p}^{+}\right]$or $\left[C_{p}^{-}\right]$forms a basis for the $S$-module $A_{T}^{*}(Y)$, and expressing the elements $\alpha$ and $\beta$ in these two bases proves sufficiency.

Combining Proposition 5.6 with Proposition 5.4 and Theorem 5.5 gives the criterion for membership in $i^{*} A_{T}^{*}(X)$ :

Theorem 5.7. A class $\alpha=\left(\alpha_{p}\right)_{p \in X^{T}} \in\left(S_{\mathbb{Q}}\right)^{X^{T}}$ lies in the image $i^{*} A_{T}^{*}(X)_{\mathbb{Q}}$ of the localization map if and only if for all $Y=X^{T^{\prime}}$ for $T^{\prime}$ a codimension one subtorus of $T$ we have

$$
\sum_{p \in Y^{T}} \frac{\alpha_{p} \beta_{p}}{e_{p}^{T}(Y)} \in S_{\mathbb{Q}},
$$

for all $\beta$ in a set of $S_{\mathbb{Q}}$-module generators for $i_{T^{\prime}}^{*} A_{T}^{*}(Y)_{\mathbb{Q}}$.

If the tangent weights of $X$ are almost coprime, the same statement holds over $\mathbb{Z}$.

Remark. When $X$ is smooth, the relations in Theorem 5.7 can also be taken for $Y$ running over all irreducible components of the union of the fixed points and the $T$-invariant curves, since such $Y$ are just the connected components of the $T^{\prime}$-fixed loci $X^{T^{\prime}}$ for some codimension one subtorus $T^{\prime}$ of $T$. We call this union of fixed points and $T$-invariant curves the one-skeleton of $X$. 
To apply Theorem 5.7, we need to know explicit generators of $A_{T}^{*}(Y)$, or more precisely their localizations to $Y^{T}$. By Proposition 5.1, one class of generators are the equivariant fundamental cycles $[Z]$ of $T$-invariant subvarieties $Z$ of the components $Y$. These are easy to compute when $Z$ is smooth, since if $p \in Z^{T}$ we have

$$
[Z]_{p}=e_{p}^{T}\left(N_{Z} Y\right),
$$

the equivariant Euler class of the normal bundle to $Z$ in $Y$, while if $p \in Y^{T} \backslash Z^{T}$, then $[Z]_{p}=0$. It follows that

$$
\frac{[Z]_{p}}{e_{p}^{T}(Y)}=\frac{1}{e_{p}^{T}(Z)}
$$

if $p \in Z^{T}$.

To see this, note that $[Z]_{p} \in A_{T}^{*}(p)$ is the pullback of $[Z]$ along the regular embedding $i_{p, Y}: p \rightarrow Y$. We factor $i_{p, Y}$ as the composition

$$
p \stackrel{i_{p, Z}}{\longrightarrow} Z \stackrel{i_{Z}}{\longrightarrow} Y \text {. }
$$

The class $[Z] \in A_{T}^{*}(Y)$ is the pushforward along $i_{Z}$ of the unit class

$$
1=[Z] \in A_{T}^{*}(Z),
$$

and so we have

$$
[Z]_{p}=i_{p, Z}^{*} i_{Z}^{*} i_{Z, *} 1=i_{p, Z}^{*} e^{T}\left(N_{Z} Y\right)=e_{p}^{T}\left(N_{Z} Y\right),
$$

by the self-intersection formula for Chow rings.

Thus if we can find for each $Y$ a collection $\mathscr{E}_{Y}$ of smooth $T$-invariant subvarieties of $Y$ so that the classes [Z] for $Z \in \mathscr{L}_{Y}$ generate $A_{T}^{*}(Y)$ as an $S$-module, we get the more explicit version of Theorem 5.7:

Theorem 5.8. A class $\alpha=\left(\alpha_{p}\right)_{p \in X^{T}} \in\left(S_{\mathbb{Q}}\right)^{X^{T}}$ lies in $i^{*} A_{T}^{*}(X)_{\mathbb{Q}}$ if and only if

$$
\sum_{p \in Z^{T}} \frac{\alpha_{p}}{e_{p}^{T}(Z)} \in S_{\mathbb{Q}},
$$

for all $Z \in \mathscr{L}_{Y}$ and all components $Y$ of the one-skeleton of $X$. If the tangent weights of $X$ are almost coprime, the same statement holds over $\mathbb{Z}$.

The necessity of (5-2) does not require the argument above, since if $\left(\alpha_{p}\right)=i_{*} \alpha$ for $\alpha \in A_{T}^{*}(X)$, then the sum is just $\pi_{*}\left(\left.\alpha\right|_{Z}\right)$, where $\pi$ is the projection of $Z$ to a point.

Obvious candidates for the subvarieties $[Z]$ are the closures of the BiałynickiBirula cells, since their classes form an $S$-basis for $A_{T}^{*}(Y)$. Unfortunately, they are not in general smooth - this was the case for Evain. However, for the quot 
schemes we study, they are smooth, as the connected components $Y$ are products of projective spaces. More generally we can ask that for each component $Y$ of $X^{T^{\prime}}$ there is a torus $T_{Y}$ containing $T$ which acts on $Y$ with finitely many orbits, so that $Y$ is a smooth toric variety. The closures of the cells will be $T_{Y}$-orbit closures, and therefore smooth.

The relations of Theorem 5.8 are the same as those found by Goldin and Holm [2001] for equivariant cohomology of Hamiltonian $T$-spaces in the case where the spaces $X^{T^{\prime}}$ are at most four-dimensional (over $\left.\mathbb{R}\right)$.

5D. Evain's relations as differential operators. We rewrite this algebraic criterion in a different form. Suppose that $Y$ is a component of $X^{T^{\prime}}$ and $Z \subset Y$ is a smooth $T$-invariant subvariety. The action of $T$ on $Y$ factors through a character $\eta: T \rightarrow \mathbb{C}^{*}$, so the weights of $T$ on $T_{p} Z$ for $p \in Z^{T}$ are nonzero scalar multiples of $\eta$. Thus there exist numbers $d_{p}=d_{p}(Z)$ so that

$$
e_{p}^{T}(Z)=d_{p}(Z) \cdot \eta^{\operatorname{dim} Z} .
$$

The terms in (5-2) have a common denominator $\eta^{\operatorname{dim} Z}$, and so we may rewrite it as

$$
\sum_{p \in Z^{T}} \frac{\alpha_{p}}{d_{p}(Z)} \in \eta^{\operatorname{dim} Z} S_{\mathbb{Q}}
$$

We can rewrite this condition using a linear differential operator. The ring $S_{\mathbb{Q}}$ is the symmetric algebra of $\hat{T}_{\mathbb{Q}}$, or dually the ring of polynomial functions on $\hat{T}_{\mathbb{Q}}^{*}$. Choose $\zeta \in \hat{T}_{\mathbb{Q}}^{*}$ for which $\zeta(\eta) \neq 0$. Then the operator $D=D_{\zeta}$ of differentiation in the direction of $\zeta$ acts on $S_{\mathbb{Q}}$. If $f \in S_{\mathbb{Q}}$ is divisible by $\eta$, then $\eta^{k}$ divides $f$ if and only if $\eta^{k-1}$ divides $D f$, so the relation (5-2) is equivalent to

$$
\sum_{p \in Z^{T}} d_{p}(Z)^{-1} D^{j} \alpha_{p} \equiv 0 \bmod \eta
$$

for all $0 \leq j<\operatorname{dim} Z$.

We give a variant of Theorem 5.8 which uses the last relation, but only with the maximum order derivative $j=\operatorname{dim} Z-1$. In exchange, we must apply it using more subvarieties $Z$.

Suppose that $Y$ is a smooth component of the one-skeleton of $X$, let $\eta$ be the associated character of $T$, and consider the two Białynicki-Birula cell decompositions

$$
\left\{C_{p}^{-} \mid p \in Y^{T}\right\}, \quad \text { and } \quad\left\{C_{p}^{+} \mid p \in Y^{T}\right\}
$$

induced by the $T$-action. Each cell $C=C_{p}^{+}, C_{p}^{-}$is isomorphic to the $T$-vector space $T_{p} C \subset T_{p} Y$. Suppose that within each cell $C=C_{p}^{+}$we can find $T$-invariant affine subspaces $C_{p, 1}, \ldots C_{p, \operatorname{dim} C}$ with $\operatorname{dim} C_{p, i}=i$ and which have smooth closures 
$Z_{p, i}=\overline{C_{p, i}}$. As before, this will be true if each $Y$ is a toric variety for a larger torus $T_{Y}$ containing $T$, since we can take each $Z_{p, i}$ to be the closure of a $T_{Y}$-orbit.

Theorem 5.9. With these assumptions, a class $\alpha=\left(\alpha_{p}\right)_{p \in X^{T}} \in\left(S_{\mathbb{Q}}\right)^{X^{T}}$ lies in $i^{*} A_{T}^{*}(X)_{\mathbb{Q}}$ if and only if

$$
\sum_{q \in Z^{T}} d_{q}(Z)^{-1} D^{\operatorname{dim} Z-1} \alpha_{q} \equiv 0 \bmod \eta,
$$

for all $Z=Z_{p, i}$ and for all components $Y$ of the one-skeleton of $X$.

Proof. The necessity of the conditions (5-3) follows from the previous discussion.

To show they are sufficient, let $U$ be an open union of the cells $C_{p}^{-}$and note that $Z_{p, i} \subset U$ if and only if $p \in U$. We use induction on the number of cells in $U$ to show that the image of $i_{U}^{*}: A_{T}^{*}(U)_{\mathbb{Q}} \rightarrow A_{T}^{*}\left(U^{T}\right)_{\mathbb{Q}}$ is the set $\left.\left(\alpha_{x}\right)\right|_{x \in U^{T}}$ satisfying (5-3) for all $Z_{p, i} \subset U$.

When $U$ is a single cell, this is immediate, as $i_{U}^{*}$ is an isomorphism and there are no $Z_{p, i}$ 's contained in $U$. Otherwise, suppose $\alpha=\left.\left(\alpha_{x}\right)\right|_{x \in U^{T}}$ satisfies (5-3) for all $Z_{p, i} \subset U$. Let $C_{p}^{-} \subset U$ be a closed cell, and put $U^{\prime}=U \backslash C_{p}^{-}$. There is an exact sequence[Brion 1997, Proposition 3.2]

$$
0 \rightarrow A_{T}^{*}(U)_{\mathbb{Q}} \stackrel{\rho}{\rightarrow} A_{T}^{*}\left(U^{\prime}\right)_{\mathbb{Q}} \times A_{T}^{*}\left(C_{p}^{-}\right)_{\mathbb{Q}} \rightarrow A_{T}^{*}\left(C_{p}^{-}\right)_{\mathbb{Q}} /\left(e^{T}(N)\right) \rightarrow 0,
$$

where $e^{T}(N)$ is the equivariant Euler class of the normal bundle $N$ to $C_{p}^{-}$in $X$. Under the isomorphism $A_{T}^{*}\left(C_{p}^{-}\right) \cong S$, this is just the product of all the $T$-weights of $N$. The components of $\rho$ are the restriction maps, while the map

$$
A_{T}^{*}\left(C_{p}^{-}\right)_{\mathbb{Q}} \rightarrow A_{T}^{*}\left(C_{p}^{-}\right)_{\mathbb{Q}} /\left(e^{T}(N)\right)
$$

is the natural quotient.

By the inductive hypothesis, $\left.\alpha\right|_{\left(U^{\prime}\right)^{T}}$ lies in the image of $i_{U^{\prime}}^{*}$. The map $A_{T}^{*}(U) \rightarrow$ $A_{T}^{*}\left(U^{\prime}\right)$ is surjective and so we can write $\alpha=i_{U}^{*} \beta+\gamma$, with $\beta \in A_{T}^{*}(U)$ and $\left.\gamma\right|_{\left(U^{\prime}\right)^{T}}=0$. Since $i_{U}^{*} \beta$ satisfies the relations (5-3), so does $\gamma$. It will be enough to show that $\gamma$ is in the image of $i_{U}^{*}$. But using the exact sequence (5-4), we see that this holds if and only if $\gamma_{p}$ is a multiple of $c_{d}^{T}(N)$, which is a nonzero multiple of $\eta^{d}$, where $d=\operatorname{codim} C_{p}^{-}=\operatorname{dim} C_{p}^{+}$. But the relation (5-3) implies that $D^{k} \gamma_{p} \equiv 0$ $(\bmod \eta)$ for $0 \leq k<d$. The result follows.

Example 5.10. Let $T=\mathbb{G}_{m}$ act on $X=\mathbb{P}^{r}$ by

$$
t \cdot\left[x_{0}: x_{1}: \cdots: x_{r}\right]=\left[x_{0}: t x_{1}: \cdots: t^{r} x_{r}\right]
$$

in homogeneous coordinates, where $t \in T$. For each $0 \leq j \leq r$, let $p_{j} \in X^{T}$ denote the $T$-fixed point corresponding to the $j$-th standard basis vector $\mathbf{e}_{j}$. The tangent 
space $T_{p_{j}} X$ is $\mathbb{C}^{n} / \mathbb{C} \cdot \mathbf{e}_{j}$, with the action of $T$ given by $t \cdot \overline{\mathbf{e}}_{k}=t^{k-j} \overline{\mathbf{e}}_{k}$. Thus

$$
e_{p_{j}}(X)=(-1)^{j} j !(r-j) ! \eta^{r},
$$

where $\eta$ is the identity character. More generally, if $0 \leq l \leq n \leq r$, let

$$
Z_{l, m}=\mathbb{P} \operatorname{Span}\left\{\mathbf{e}_{l}, \mathbf{e}_{l+1}, \ldots, \mathbf{e}_{m}\right\} .
$$

The same calculation gives

$$
e_{p_{j}}\left(Z_{l, m}\right)=(-1)^{j-l}(j-l) !(m-j) ! \eta^{m-l} .
$$

We can apply Theorem 5.8 using the smooth subvarieties $Z_{0, l}$, for $1 \leq l \leq r$. Then $i^{*} A_{T}^{*}(X) \subset A_{T}^{*}\left(X^{T}\right)$ is the set of tuples $\alpha=\left(\alpha_{0}, \ldots, \alpha_{r}\right)$ where

$$
\sum_{0 \leq j \leq l} \frac{(-1)^{j} \alpha_{j}}{j !(l-j) !} \in \eta^{l} S
$$

for all $1 \leq l \leq r$.

On the other hand, we can apply Theorem 5.9 using all the subvarieties $Z_{l, m}$. If $D$ is differentiation on $S$ in the direction of $\eta^{\vee}$, then $\alpha$ lies in the image of $i^{*}$ if and only if

$$
\sum_{l \leq j \leq m} \frac{(-1)^{j-l} D^{m-l-1} \alpha_{j}}{(j-l) !(m-j) !} \in \eta S,
$$

for all $0 \leq l<m \leq r$. We could take one more derivative and ask that the resulting sums vanish, but this would not generalize to actions of higher-dimensional tori.

When $X=\mathbb{P}^{1}$ we get exactly the GKM relation for a primitive action.

Example 5.11. These same arguments apply to products of projective spaces. Let $\mathbf{r}=\left(r_{1}, \ldots, r_{n}\right)$, and let $X=\mathbb{P}^{r_{1}} \times \cdots \times \mathbb{P}^{r_{n}}$, where the action of $t \in T=\mathbb{G}_{m}$ on a point $\left(\left[x_{0}^{1}: x_{1}^{1}: \cdots: x_{r_{1}}^{1}\right], \ldots,\left[x_{0}^{n}: x_{1}^{n}: \cdots: x_{r_{n}}^{n}\right]\right)$ is given by multiplying $x_{j}^{i}$ by $t^{j}$. The fixed points have the form $p_{\mathbf{j}}=\left(p_{j_{1}}, \ldots, p_{j_{n}}\right)$, where $p_{j_{i}}$ is the $j_{i}$-th fixed point in $\mathbb{P}^{r_{i}}$, in the notation of Example 5.10, and $\mathbf{j}=\left(j_{1}, \ldots, j_{n}\right)$ satisfies $0 \leq \mathbf{j} \leq \mathbf{r}$, meaning that $0 \leq j_{i} \leq r_{i}$ for all $1 \leq i \leq n$.

For $\mathbf{l}, \mathbf{m} \in \mathbb{Z}^{n}$ with $0 \leq l_{i} \leq m_{i} \leq r_{i}$ for all $i$, set $Z_{\mathbf{l}, \mathbf{m}}=Z_{l_{1}, m_{1}} \times \cdots \times Z_{l_{n}, m_{n}}$. For each $\mathbf{l} \leq \mathbf{j} \leq \mathbf{m}$, the tangent space to $Z_{\mathbf{l}, \mathbf{m}}$ at $p_{\mathbf{j}}$ is $\bigoplus_{i=1}^{n} T_{p_{j_{i}}} Z_{l_{i}, m_{i}}$. Using the computation from Example 5.10, we see that

$$
e_{p_{\mathbf{j}}}^{T}\left(Z_{\mathbf{l}, \mathbf{m}}\right)=(-1)^{|\mathbf{j}|}(\mathbf{j}-\mathbf{l}) !(\mathbf{m}-\mathbf{j}) ! \eta^{|\mathbf{m}|-|\mathbf{l}|} .
$$

Recall that for an $n$-tuple $\mathbf{a}=\left(a_{1}, \ldots, a_{n}\right)$, we put $\mathbf{a} !=a_{1} ! \cdots a_{n} !$.

As in Example 5.10, we can either apply Theorem 5.8 using the subvarieties $Z_{\mathbf{0}, \mathbf{l}}$, or Theorem 5.9 using all the $Z_{\mathbf{l}, \mathbf{m}}$. The resulting conditions for a tuple $\alpha=$ 
$\left(\alpha_{\mathbf{j}}\right)_{\mathbf{0} \leq \mathbf{j} \leq \mathbf{r}}$ to be in the image of the localization map are just (5-5) and (5-6), where the variables now represent elements of $\mathbb{Z}^{n}$ rather than scalars.

\section{E. Proof of Theorem 1.1.}

Proof. We combine these localization results with the geometry of the quot scheme from Sections 2, 3, and 4 to produce a proof of Theorem 1.1.

The only nonprimitive tangent weights are those with $i=j$ in (2-3), which are multiples of $\mathbf{f}$. These correspond to Type III curves. Thus the tangent weights are almost coprime, so Theorem 5.8 gives a correct description of the integral equivariant Chow ring.

The relations I, II(a) and II(b) are the GKM relations for the $T$-invariant curves of types I and II, as described in Section 3 using the identification of the $T$-weights of these curves with the tangent weights given by Theorem 3.2.

The relations $\mathbf{I I}(\mathrm{c}) / \mathbf{I I}(\mathrm{c})^{\prime}$ come from the horizontal families of $T$-invariant curves of type II. As described in Section 4, these are isomorphic to $\mathbb{P}^{1} \times \mathbb{P}^{1}$, where the action on each factor is by the same primitive character. As in Example 5.11, we can apply the relation in Theorem 5.8 with $Y=\mathbb{P}^{1} \times \mathbb{P}^{1}$ to get the relations II(c)/II(c)'. We get one new relation using $Z=Y$; smaller $T$-invariant subvarieties contained in $Y$ are either $T$-invariant curves, whose relations are already covered by $\mathbf{I I}(\mathrm{a})$ and $\mathbf{I I}(\mathrm{b})$, or points, which give no relation.

Finally, the relations III and III' come from the vertical families. As described in Section 4 , the vertical family containing $\mathscr{S}_{(\boldsymbol{\delta}, \mathbf{a}, \mathbf{b})}$ is isomorphic to

$$
\prod_{\delta_{i}=1} \mathbb{P} H^{0}\left(\mathbb{O}\left(a_{i}+b_{i}\right)\right) \simeq \prod_{\delta_{i}=1} \mathbb{P}^{a_{i}+b_{i}},
$$

and the fixed points in the family are those $\mathscr{S}_{\left(\delta, \mathbf{a}^{\prime}, \mathbf{b}^{\prime}\right)}$ with $\mathbf{a}^{\prime}+\mathbf{b}^{\prime}=\mathbf{a}+\mathbf{b}$. The codimension one subtorus $T_{k^{n}} \subset T$ acts trivially on the family, and the remaining action of $T_{\mathbb{P} 1}$ is the one described in Example 5.11, using the monomial basis of $H^{0}\left(\mathcal{O}\left(a_{i}+b_{i}\right)\right)$. Example 5.11 then gives exactly the relations III and III'. This proves Theorem 1.1.

\section{Equivariant Chern classes on $2_{d}$}

Recall that $\mathbb{P}^{1} \times 2_{d}$ has a universal exact sequence of sheaves

$$
0 \rightarrow \mathscr{S} \rightarrow \mathbb{O}_{\mathbb{P} 1 \times \mathscr{Q}_{d}}^{n} \rightarrow \mathscr{T} \rightarrow 0
$$

with $\mathscr{Y}$ the tautological vector bundle of rank $n-r$.

Since both $2_{d}$ and $\mathbb{P}^{1}$ have cell decompositions, we have a Künneth decomposition of Chow rings,

$$
A^{*}\left(\mathbb{P}^{1} \times \mathscr{2}_{d}\right) \simeq A^{*}\left(\mathbb{P}^{1}\right) \otimes_{\mathbb{Z}} A^{*}\left(\mathscr{2}_{d}\right) .
$$


Let $\pi: \mathbb{P}^{1} \times 2_{d} \rightarrow 2_{d}$ be the projection. For each $1 \leq i \leq n-r$ we may decompose the Chern class $c_{i}(\mathscr{Y})$ as

$$
c_{i}(\mathscr{S})=\pi^{*} t_{i}+h \pi^{*} u_{i-1},
$$

where $t_{i}, u_{i} \in A^{i}\left(2_{d}\right)$ and $h$ is the class of a point in $A^{1}\left(\mathbb{P}^{1}\right)$ pulled back to $\mathbb{P}^{1} \times 2_{d}$. Note that $u_{0}=-d$. Strømme [1987] proved that $A^{*}\left(2_{d}\right)_{\mathbb{Q}}$ is generated by the classes

$$
\left\{t_{1}, \ldots, t_{k}, u_{1}, \ldots, u_{k-1}\right\} .
$$

For each $1 \leq i \leq n-r$, the equivariant Chern class $c_{i}^{T}(\mathscr{S})$ localizes at a fixed point $p$ to the $i$-th elementary symmetric polynomial $e_{i}$ in the $T$-weights of the fibre of $\mathscr{Y}$ at $p$. The fixed points of $\mathbb{P}^{1} \times \mathscr{2}_{d}$ correspond to $\{0, \infty\} \times \mathscr{F}_{\text {. For }}(\boldsymbol{\delta}, \mathbf{a}, \mathbf{b}) \in \mathscr{F}$, the bundle $\mathscr{Y}_{(\boldsymbol{\delta}, \mathbf{a}, \mathbf{b})}$ on $\mathbb{P}^{1}$ is a sum of line bundles $\mathscr{Y}_{a_{j}, b_{j}} \mathbf{e}_{j}$ for $\delta_{j}=1$. Since $\mathscr{S}_{a, b}$ has weight $a \mathbf{f}$ at 0 and $-b \mathbf{f}$ at $\infty$, the localizations of $c_{i}^{T}(\mathscr{Y})$ are

$$
\begin{array}{cl}
c_{i, 0,(\boldsymbol{\delta}, \mathbf{a}, \mathbf{b})}^{T}=e_{i}\left(\left\{\mathbf{e}_{j}+a_{j} \mathbf{f} \mid \delta_{j}=1\right\}\right) & \text { at }(0,(\boldsymbol{\delta}, \mathbf{a}, \mathbf{b})), \\
c_{i, \infty,(\boldsymbol{\delta}, \mathbf{a}, \mathbf{b})}^{T}=e_{i}\left(\left\{\mathbf{e}_{j}-b_{j} \mathbf{f} \mid \delta_{j}=1\right\}\right) & \text { at }(\infty,(\boldsymbol{\delta}, \mathbf{a}, \mathbf{b})) .
\end{array}
$$

We also have a Künneth decomposition in equivariant Chow cohomology:

$$
A_{T}^{*}\left(\mathbb{P}^{1} \times \mathscr{2}_{d}\right) \stackrel{\sim}{\longleftarrow} A_{T}^{*}\left(\mathbb{P}^{1}\right) \otimes_{S} A_{T}^{*}\left(\mathscr{Q}_{d}\right) .
$$

To see this, just imitate the argument for ordinary Chow cohomology; the equivariant Chow cohomology of a variety with an algebraic cell decomposition will be a free $S$-module, with a module basis given by the closures of the cells.

An equivariant Künneth decomposition of $c_{i}^{T}(\mathscr{S})$ analogous to (6-1) requires the choice of a lift of the class of a point to $A_{T}^{1}\left(\mathbb{P}^{1}\right)$. Localizing a class $x \in A_{T}^{1}\left(\mathbb{P}^{1}\right)$ gives an ordered pair $\left(x_{0}, x_{\infty}\right) \in \mathbb{Z} \oplus \oplus \mathbb{Z} \mathbf{f}$. Lifts of classes from $A^{1}\left(\mathbb{P}^{1}\right)$ are only well-defined modulo the span of (f, f). Three possible choices for lifting the class of a point are

$$
\text { (i) }(-\mathbf{f}, 0), \quad(\text { ii })(0, \mathbf{f}), \quad \text { and } \quad \text { (iii) } \frac{1}{2}(-\mathbf{f}, \mathbf{f}) \text {. }
$$

The symmetric lift ( $\mathrm{iii}$ ) requires rational coefficients. We will use this lift to express our formulas.

Given a lift $h \in A_{T}^{1}\left(\mathbb{P}^{1}\right)$ of the class of a point, the formula (6-1) defines equivariant lifts of the classes $t_{i}, u_{i-1}$. Strømme's result together with Proposition 5.2 implies that these classes generate $A_{T}^{*}(X)_{\mathbb{Q}}$ as an $S_{\mathbb{Q}}$-algebra.

Proposition 6.1. The symmetric choice (iii) of lift $h \in A_{T}^{1}\left(\mathbb{P}^{1}\right)$ of the class of a point gives the following formula for the Künneth components $t_{i}, u_{i-1}$ of the equivariant Chern class $c_{i}^{T}(\mathscr{Y})$ of the bundle $\mathscr{Y}$, expressed in terms of their localizations 
at the fixed point of $2_{d}$ indexed by $p:=(\boldsymbol{\delta}, \mathbf{a}, \mathbf{b})$ :

$$
t_{i, p}=\frac{1}{2}\left(c_{i, 0, p}^{T}+c_{i, \infty, p}^{T}\right), \quad \text { and } \quad u_{i-1, p}=\frac{1}{\mathbf{f}}\left(c_{i, \infty, p}^{T}-c_{i, 0, p}^{T}\right) .
$$

\section{Equivariant Chow ring of the quot scheme $2_{2}(0,2)$}

We use Theorem 1.1 to describe the equivariant Chow ring of $2_{2}:=2_{2}(0,2)$. We first give a basis for $A_{T}^{*}\left(\mathscr{2}_{2}\right)$ as a module over $S=\mathbb{Z}\left[\mathbf{e}_{1}, \mathbf{e}_{2}, \mathbf{f}\right]$.

The equivariant Chow ring is the collection of tuples $\left(f_{p} \mid p \in 2_{2}^{T}\right) \in S_{2}^{2_{2}^{T}}$ which satisfy the relations of Theorem 1.1. If $p$ and $q$ are connected by an edge in the moment multigraph (see Figure 1) with weight $\chi$, then $f_{p}-f_{q}$ lies in the ideal generated by $\chi$. These are the standard GKM relations.

There are two multiedges with four vertices in the multigraph, namely the vertical and horizontal lines of symmetry. They should be seen as flattened quadrangles, since they are images of subvarieties isomorphic to $\mathbb{P}^{1} \times \mathbb{P}^{1}$. Each gives rise to an additional relation as follows. Suppose that the quadrangle has four vertices $a, b, c, d$ :

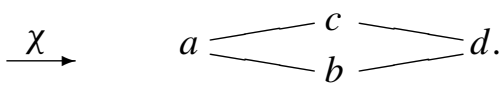

(Here, the edges are parallel with direction $\chi$.) Then the tuples $\left(f_{p}\right)$ must satisfy

$$
f_{a}-f_{b}-f_{c}+f_{d} \in \chi^{2} S .
$$

These are relations of types II(c) (horizontal) and III' (vertical) of Theorem 1.1.

The remaining multiedges with more than two vertices are the left and right vertical edges, both with three vertices. They should be seen as flattened triangles, since they come from subvarieties isomorphic to $\mathbb{P}^{2}$. The additional relations they induce are described as follows. Let the three fixed points on the multiedge be $a, b, c$, with $b$ between $a$ and $c$. Then

$$
\frac{1}{2} f_{a}-f_{b}+\frac{1}{2} f_{c} \in \mathbf{f}^{2} S .
$$

This is relation of type III' of Theorem 1.1.

When $k=\mathbb{C}$, we can construct an $S$-module basis for equivariant cohomology using equivariant Morse theory; as is well-known, a generic projection of the moment map to a line will give a Morse function which is perfect for equivariant cohomology. This results in an inductive algorithm to produce a basis, which is nicely expounded in [Tymoczko 2005] (see also [Guillemin and Zara 2001]). Pick a direction vector $\mathbf{v}$ (corresponding to an element of the Lie algebra $\mathfrak{t}_{\mathbb{R}}$ ) which does not annihilate the direction vector of any edge. Orient each edge to have positive pairing with $\mathbf{v}$; this is the Hasse diagram of a partial order on the fixed points induced by $\mathbf{v}$. Then, using the relations described above, we can inductively 

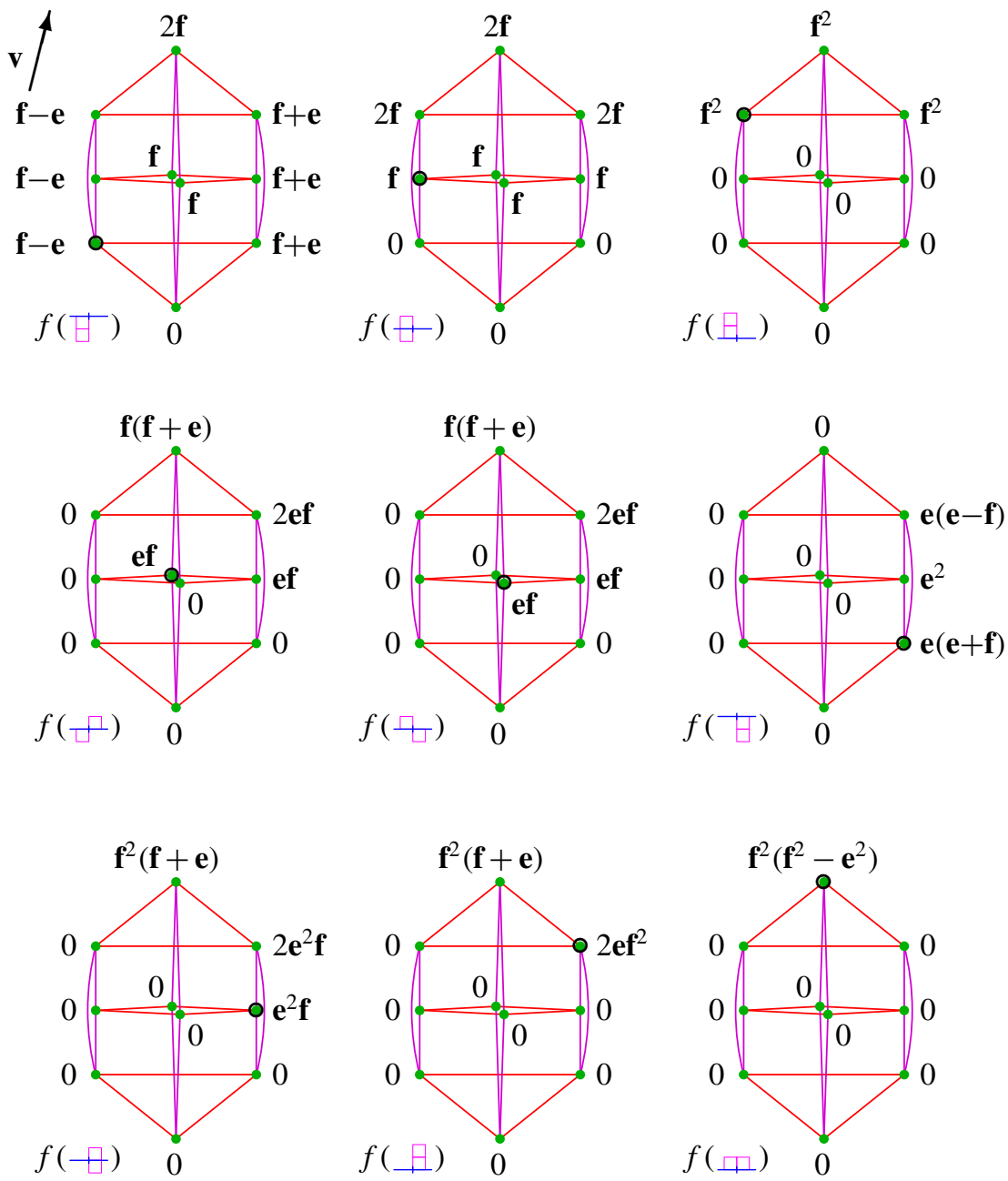

Figure 2. An $S$-module basis for $A_{T}^{*}\left(2_{2}(0,2)\right)$.

construct a triangular basis with respect to this ordering. That is, if $f=f(p)$ corresponds to the fixed point $p$, it vanishes at $q\left(f_{q}=0\right)$ unless $p<q$, and $f_{p}$ is the product of weights of edges pointing down from $p$. While this algorithm was motivated by Morse theory, it makes sense over any field $k$, if $\mathbf{v}$ is a linear function on the character group of $T$ which does not annihilate any edge of the moment graph.

Set $\mathbf{e}:=\mathbf{e}_{1}-\mathbf{e}_{2}$ and pick the vector $\mathbf{v}=\mathbf{f}+\epsilon \mathbf{e}$, where $\epsilon>0$ is small. One basis element is the identity $f(\amalg)$, which localizes to 1 at each fixed point. We display each of the remaining nine in Figure 2 as a localization diagram, writing its localizations on a copy of a moment multigraph. 

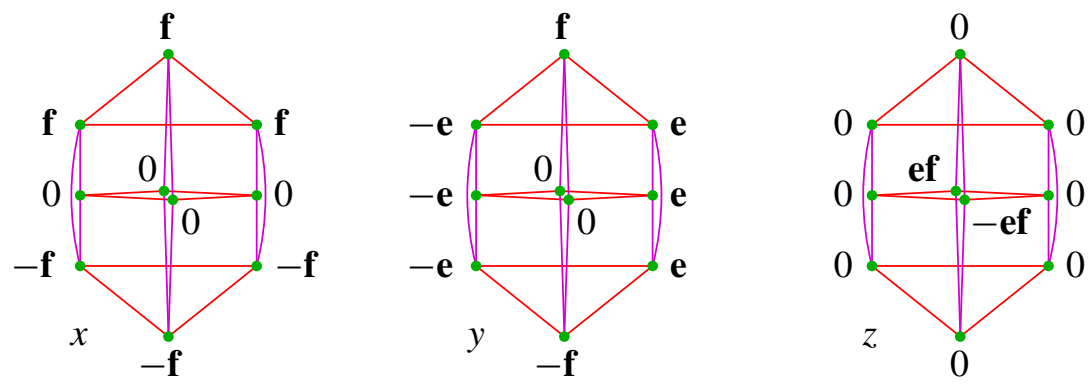

Figure 3. Generators for the $S_{\mathbb{Q}^{-}}$-algebra $A_{T}^{*}\left(2_{2}(0,2)\right)_{\mathbb{Q}}$.

Set $x:=f(\square)-\mathbf{f}, y:=f(\square)-\mathbf{f}$, and $z:=f(\square)-f(\square)$. Figure 3 shows their localization diagrams. We show that they generate $A_{T}^{*}\left(2_{2}\right)_{\mathbb{Q}}$ as an $S_{\mathbb{Q}^{-}}$ algebra by showing that each basis element $f(p)$ of degree greater than 2 lies in $S_{\mathbb{Q}}[x, y, z]$. Since

$$
\begin{aligned}
f(\boxminus) & =\frac{1}{2} x(x+\mathbf{f}), \\
f(\square) & =\frac{1}{2}(y+\mathbf{e})(y-x), \text { and } \\
f(\square)+f(\square) & =(y+\mathbf{e})(x+\mathbf{f}),
\end{aligned}
$$

the four degree 2 basis elements lie in $S_{\mathbb{Q}}[x, y, z]$. The remaining three basis elements also lie in $S_{\mathbb{Q}}[x, y, z]$,

$$
y f(\square)=f(\square), \quad x f(\square)=f(\not), \quad \text { and } \quad(y-\mathbf{e}) f(\square)=f(\square) .
$$

Inspecting the localization diagrams of $x, y$, and $z$ shows that the following 5 expressions vanish in $A_{T}^{*}\left(2_{2}\right)$ :

$$
x z, y z, x\left(x^{2}-\mathbf{f}^{2}\right),\left(y^{2}-\mathbf{e}^{2}\right)(y-x), \text { and } z^{2}-\left(y^{2}-\mathbf{e}^{2}\right)\left(x^{2}-\mathbf{f}^{2}\right) .
$$

In the lexicographic term order where $z>y>x>e>f$, these five polynomials form a Gröbner basis for the ideal $\mathscr{I}$ of $\mathbb{Q}\left[\mathbf{e}_{1}, \mathbf{e}_{2}, \mathbf{f}, x, y, z\right]$ they generate with leading terms $x z, y z, x^{3}, y^{3}$, and $z^{2}$. There are ten standard monomials

$$
1, x, y, x^{2}, x y, y^{2}, z, x^{2} y, x y^{2} \text {, and } x^{2} y^{2} .
$$

Since $A_{T}^{*}\left(2_{2}\right)_{\mathbb{Q}}$ is free over $S_{\mathbb{Q}}=\mathbb{Q}\left[\mathbf{e}_{1}, \mathbf{e}_{2}, \mathbf{f}\right]$ of rank 10 , we conclude that

$$
A_{T}^{*}\left(2_{2}\right)_{\mathbb{Q}} \simeq \mathbb{Q}\left[\mathbf{e}_{1}, \mathbf{e}_{2}, \mathbf{f}, x, y, z\right] / \mathscr{\Phi} .
$$

Using Proposition 5.2, we obtain the presentation of the rational Chow ring

$$
A^{*}\left(2_{2}\right)_{\mathbb{Q}} \simeq \mathbb{Q}[x, y, z] /\left\langle x z, y z, x^{3}, x y^{2}-y^{3}, z^{2}-x^{2} y^{2}\right\rangle .
$$




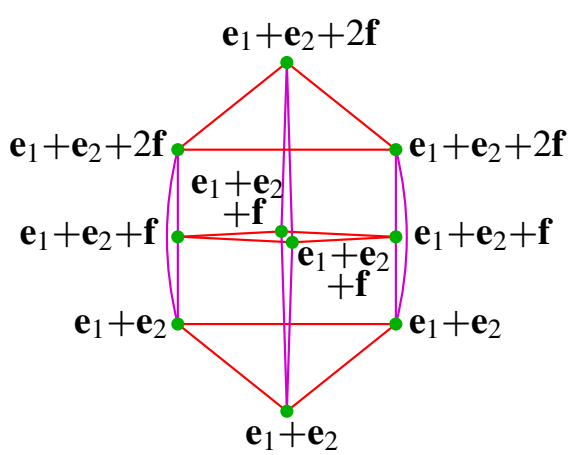

Localization of $c_{1}^{T}(\mathscr{S})$ at $0 \in \mathbb{P}^{1}$

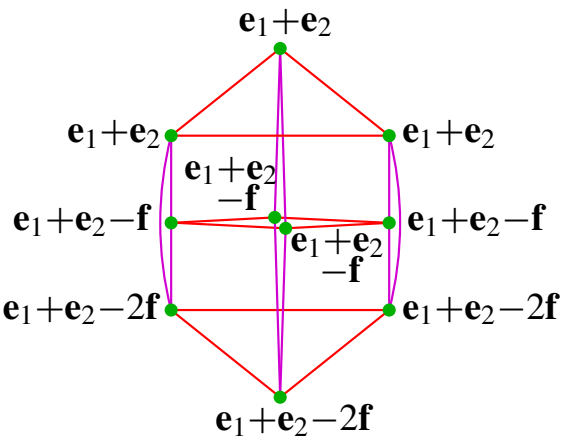

Localization of $c_{1}^{T}(\mathscr{S})$ at $\infty \in \mathbb{P}^{1}$

Figure 4. First Chern class of $\mathscr{S}$.

Looking at (7-1), we see that the integral Chow ring is not generated by $x, y$, and $z$, so its presentation will be considerably more complicated.

We now consider Strømme's generators. Figure 4 shows the localization diagram of the first Chern class of $\mathscr{Y}$. By the formula of Proposition 6.1, we have

$$
c_{1}^{T}(\mathscr{S})=\mathbf{e}_{1}+\mathbf{e}_{2}+x-2 h,
$$

so that

$$
t_{1}=\mathbf{e}_{1}+\mathbf{e}_{2}+x, \quad \text { and } \quad u_{0}=-2 .
$$

Figure 5 shows the localization diagram of the second Chern class of $\mathscr{Y}$. By the formula of Proposition 6.1, we have

$$
c_{2}^{T}(\mathscr{S})=\mathbf{e}_{1} \mathbf{e}_{2}+\frac{1}{2} x\left(y+\mathbf{e}_{1}+\mathbf{e}_{2}\right)+\frac{1}{2} z-h\left(y+\mathbf{e}_{1}+\mathbf{e}_{2}\right),
$$

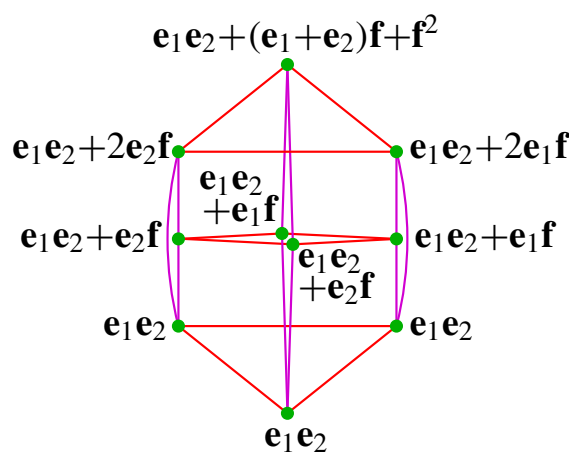

Localization of $c_{2}^{T}(\mathscr{S})$ at $0 \in \mathbb{P}^{1}$

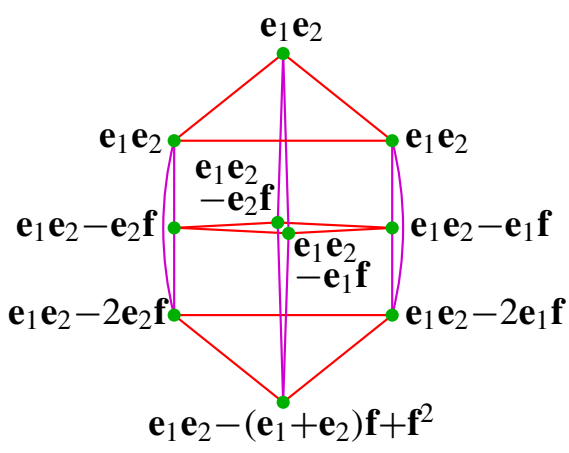

Localization of $c_{2}^{T}(\mathscr{S})$ at $\infty \in \mathbb{P}^{1}$

Figure 5. Second Chern class of $\mathscr{Y}$. 
so that

$$
t_{2}=\mathbf{e}_{1} \mathbf{e}_{2}+\frac{1}{2} x\left(y+\mathbf{e}_{1}+\mathbf{e}_{2}\right)+\frac{1}{2} z \quad \text { and } \quad u_{1}=-\left(y+\mathbf{e}_{1}+\mathbf{e}_{2}\right) .
$$

The corresponding classes in $A^{*}\left(2_{2}\right)$ are

$$
t_{1}=x, \quad u_{1}=-y, \quad \text { and } \quad t_{2}=\frac{1}{2}(z+x y) .
$$

Remark. This shows that the claim in [Strømme 1987, Theorem 5.3] that the classes $t_{i}, u_{i-1}$ generate the integral Chow ring is false.

\section{References}

[Bertram 1997] A. Bertram, "Quantum Schubert calculus", Adv. Math. 128:2 (1997), 289-305. MR 98j:14067 Zbl 0945.14031

[Białynicki-Birula 1973] A. Białynicki-Birula, "Some theorems on actions of algebraic groups", Ann. of Math. (2) 98 (1973), 480-497. MR 51 \#3186 Zbl 0275.14007

[Brion 1997] M. Brion, "Equivariant Chow groups for torus actions", Transform. Groups 2:3 (1997), 225-267. MR 99c:14005 Zbl 0916.14003

[Chang and Skjelbred 1974] T. Chang and T. Skjelbred, "The topological Schur lemma and related results", Ann. of Math. (2) 100 (1974), 307-321. MR 51 \#11552 Zbl 0288.57021

[Edidin and Graham 1998a] D. Edidin and W. Graham, "Equivariant intersection theory", Invent. Math. 131:3 (1998), 595-634. MR 99j:14003a Zbl 0940.14003

[Edidin and Graham 1998b] D. Edidin and W. Graham, "Localization in equivariant intersection theory and the Bott residue formula", Amer. J. Math. 120:3 (1998), 619-636. MR 99g:14005 Zbl 0980.14004

[Eisenbud 1995] D. Eisenbud, Commutative algebra. With a view toward algebraic geometry, Graduate Texts in Mathematics 150, Springer, New York, 1995. MR 97a:13001 Zbl 0819.13001

[Evain 2007] L. Evain, "The Chow ring of punctual Hilbert schemes on toric surfaces”, Transform. Groups 12:2 (2007), 227-249. MR 2008f:14008 Zbl 1128.14004

[Fulton 1998] W. Fulton, Intersection theory, 2nd ed., Results in Mathematics and Related Areas. 3rd Series. A Series of Modern Surveys in Mathematics 2, Springer, Berlin, 1998. MR 99d:14003 Zbl 0885.14002

[Goldin and Holm 2001] R. Goldin and T. S. Holm, "The equivariant cohomology of Hamiltonian $G$-spaces from residual $S^{1}$ actions”, Math. Res. Lett. 8:1-2 (2001), 67-77. MR 2002f:53140 Zbl 0987.57011

[Goresky et al. 1998] M. Goresky, R. Kottwitz, and R. MacPherson, "Equivariant cohomology, Koszul duality, and the localization theorem”, Invent. Math. 131:1 (1998), 25-83. MR 99c:55009 Zbl 0897.22009

[Guillemin and Zara 2001] V. Guillemin and C. Zara, "1-skeleta, Betti numbers, and equivariant cohomology”, Duke Math. J. 107:2 (2001), 283-349. MR 2002j:53110 Zbl 1020.57013

[Guillemin and Zara 2002] V. Guillemin and C. Zara, "Combinatorial formulas for products of Thom classes", pp. 363-405 in Geometry, mechanics, and dynamics, edited by P. Newton et al., Springer, New York, 2002. MR 2003i:53121 Zbl 1009.57034

[Guillemin and Zara 2003] V. Guillemin and C. Zara, "The existence of generating families for the cohomology ring of a graph", Adv. Math. 174:1 (2003), 115-153. MR 2004f:53099 Zbl 1020.57014 
[Iversen 1972] B. Iversen, "A fixed point formula for action of tori on algebraic varieties", Invent. Math. 16 (1972), 229-236. MR 45 \#8656 Zbl 0246.14010

[Strømme 1987] S. A. Strømme, “On parametrized rational curves in Grassmann varieties”, pp. 251-272 in Space curves (Rocca di Papa, 1985), edited by F. Ghione et al., Lecture Notes in Math. 1266, Springer, Berlin, 1987. MR 88i:14020 Zbl 0625.14027

[Tymoczko 2005] J. S. Tymoczko, "An introduction to equivariant cohomology and homology, following Goresky, Kottwitz, and MacPherson", pp. 169-188 in Snowbird lectures in algebraic geometry (Snowbird, UT, 2004), edited by R. Vakil, Contemp. Math. 388, Amer. Math. Soc., Providence, RI, 2005. MR 2006m:55019

Received November 28, 2007. Revised July 14, 2008.

TOM BRADEN

DEPARTMENT OF MATHEMATICS AND STATISTICS

LEDERLE GRAdUATE RESEARCH TOWER, BoX 34515

UNIVERSITY OF MASSACHUSETTS AMHERST

AMHERST, MA 01003-9305

UNITED STATES

braden@math.umass.edu

http://www.math.umass.edu/ braden/

\section{LINDA CHEN}

DEPARTMENT OF MATHEMATICS

THE OHIO STATE UNIVERSITY

231 West 18Th AVEnUe

Columbus, OH 43210-1174

UNITED STATES

lchen@math.ohio-state.edu

and

DePartment of Mathematics and Statistics

SWARTHMORE COLLEGE

SWARTHMORE, PA 19081

UNITED STATES

lchen@swarthmore.edu

FRANK SOTTILE

DEPARTMENT OF MATHEMATICS

TEXAS A\&M UNIVERSITY

College Station, TX 77843

UNITED STATES

sottile@math.tamu.edu

http://www.math.tamu.edu/ sottile/ 